\title{
On generalizing free algebras for a functor*
}

\author{
Dion Coumans and Sam van Gool ${ }^{\dagger}$
}

November 21, 2011

\begin{abstract}
In this paper we introduce a new setting, based on partial algebras, for studying constructions of finitely generated free algebras. We give sufficient conditions under which the finitely generated free algebras for a variety $\mathbf{V}$ may be described as the colimit of a chain of finite partial algebras obtained by repeated application of a functor. In particular, our method encompasses the construction of finitely generated free algebras for varieties of algebras for a functor as in [2], Heyting algebras as in [1] and S4 algebras as in [8].
\end{abstract}

\section{Introduction}

In the algebraic study of a logic $\mathcal{L}$, one assigns a class of algebras $\mathbf{V}_{\mathcal{L}}$ to the logic and uses algebraic methods to obtain properties of this class. The results of this algebraic study can be translated back to properties of $\mathcal{L}$. Algebraic methods may be applied to study issues such as term complexity, decidability of logical equivalence, interpolation and normal forms, i.e., problems in which one considers formulas whose variables are drawn from a finite set. In particular, if the class of algebras $\mathbf{V}_{\mathcal{L}}$ contains (finitely generated) free algebras, a thorough understanding of these can yield powerful results about the logic $\mathcal{L}$.

In [2] N. Bezhanishvilli and Kurz study classes of algebras $\mathbf{V}_{\mathcal{L}}$ associated with a logic $\mathcal{L}$ which is axiomatized by equations which are rank 1 for an operation $f^{1}$. In this case, the algebras for the logic can be represented as algebras for a functor $F_{\mathcal{L}}$ on the category of

\footnotetext{
*Keywords: free algebras, partial algebras, quasiequations, rank 0-1.

${ }^{\dagger}$ Institute for Mathematics, Astrophysics and Particle Physics, Radboud Universiteit Nijmegen, P.O. Box 9010, 6500 GL Nijmegen, The Netherlands,d.coumans@math.ru.nl/s.vangool@math.ru.nl. The authorswould like to thank their PhD supervisor Mai Gehrke, for proposing this project in the first place and guidance along the way. Furthermore they would like to thank the anonymous referees for their valuable suggestions. The PhD research project of the last-named author has been made possible by NWO grant 617.023.815 of the Netherlands Organization for Scientific Research (NWO).

${ }^{1}$ An equation is of rank 1 for an operation $f$ if every variable occurs under the scope of exactly one occurrence of $f$.
} 
underlying algebras without the operation $f$. The authors show that this functor $F_{\mathcal{L}}$ enables a constructive description of the free $\mathbf{V}_{\mathcal{L}}$ algebras.

As many interesting logics are not axiomatized by rank 1 axioms, one would want to extend these existing techniques. However, as is shown in [11], non-rank 1 logics cannot be represented as algebras for a functor and therefore we cannot use the standard construction of free algebras in a straightforward way.

Ghilardi pioneered the construction of free algebras for non-rank 1 varieties in [6]. Here he describes a method to incrementally build finitely generated free Heyting algebras by constructing a chain of distributive lattices, where, in each step, implications are freely added to the lattice, while keeping a specified set of implications which are already defined in the previous step. In a subsequent paper, Ghilardi extended these techniques to modal logic [7], and used his algebraic and duality theoretic methods to derive normal forms for modal logics, notably S4.

Recently, this line of research has been picked up again. In [1] N. Bezhanishvili and Gehrke have re-analysed Ghilardi's incremental construction and have described it by repeated application of a functor on the category of algebras for the logic, based on the ideas of the coalgebraic approach to rank 1 logics and Birkhoff duality for finite distributive lattices. Shortly after, Ghilardi [8] gave a new construction of the free S4 algebra in the same spirit. However, the methods in [1] and [8] rely on specific properties of Heyting algebras and S4 algebras respectively, and they do not directly apply in a general setting. Studying this work has led us to the insight that partial algebras are the natural structures to consider when building free algebras step by step. This insight has enabled us to describe a general functorial method for constructing free algebras which is applicable outside the setting of pure rank 1 logics.

\subsection{Outline}

We will now outline our method in a bit more detail. Although our method is applicable to more general logics, our focus in this paper (mainly for the sake of readability) is on modal logics, i.e., Boolean logics with one additional unary $\vee$-preserving connective $\diamond$, and their associated algebras: modal algebras. The notion of rank of a modal term is central in this paper and therefore we give a precise definition.

Definition 1.1. Let $P$ be a set of variables. We denote the set of Boolean terms in $P$ by $\mathrm{T}_{\mathbf{B A}}(P)$. The sets $\mathrm{T}_{\mathbf{M A}}^{n}(P)$ of modal terms in $P$ of rank at most $n$ are defined inductively 
as follows.

$$
\begin{aligned}
\mathrm{T}_{\mathbf{M A}}^{0}(P) & :=\mathrm{T}_{\mathbf{B A}}(P), \\
\mathrm{T}_{\mathbf{M A}}^{n+1}(P) & :=\mathrm{T}_{\mathbf{B A}}\left(P \cup\left\{\diamond t: t \in \mathrm{T}_{\mathbf{M A}}^{n}(P)\right\}\right) .
\end{aligned}
$$

Recall that an equation is an expression " $s \approx t$ ", where $s$ and $t$ are modal terms, and a modal algebra $\left(B, \diamond^{B}\right)$ is said to satisfy an equation if the interpretations of the terms $s$ and $t$ in $\left(B, \diamond^{B}\right)$ are equal, under all interpretations of the variables. Similarly, a quasiequation is an expression of the form " $\left(s_{1} \approx t_{1} \& \cdots \& s_{m} \approx t_{m}\right) \rightarrow(s \approx t)$ ", and we can express when a modal algebra satisfies a quasi-equation in the obvious way. Given a set of (quasi-)equations $\mathcal{E}$, the (quasi-)equational class $\mathbf{V}_{\mathcal{E}}$ is the class of modal algebras satisfying all (quasi-)equations in $\mathcal{E}$. A classical theorem of Birkhoff says that, for every (quasi-)equational class $\mathbf{V}$ of modal algebras and set of variables $P$, the free $\mathbf{V}$ algebra over $P, F_{\mathbf{V}}(P)$, exists.

The notion of rank allows us to understand this free algebra in a layered manner as follows. For each $n \geq 0$, the (equivalence classes of) terms of rank at most $n$ form a Boolean subalgebra $B_{n}$ of $F_{\mathbf{V}}(P)$. Furthermore, for each $n$, the operator $\diamond$ on $F_{\mathbf{V}}(P)$ yields a join preserving map $\diamond_{n+1}: B_{n} \rightarrow B_{n+1}$. Hence, we have a chain of Boolean algebras

$$
B_{0} \stackrel{\diamond_{1}}{\rightleftarrows} B_{1} \stackrel{\diamond_{2}}{\longrightarrow} B_{2} \stackrel{\diamond_{3}}{\longrightarrow} \ldots
$$

with embeddings and join-preserving maps between them. The Boolean reduct of $F_{\mathbf{V}}(P)$ is the colimit of the chain of Boolean algebras and embeddings and the operator $\diamond$ is the unique extension of the functions $\diamond_{n}$ to a function on $F_{\mathbf{V}}(P)$.

The new perspective on this chain that we propose in this paper is the following. Instead of considering $\diamond_{n+1}$ as a map $B_{n} \rightarrow B_{n+1}$, we propose to view it as a partial operator on $B_{n+1}$ (which is only defined on elements in the subalgebra $B_{n}$ ). This leads to the notion of partial modal algebra (cf. Definition 2.1) and the above chain may be described as a chain in the category of partial modal algebras

$$
\left(B_{1}, \diamond_{1}\right) \mapsto\left(B_{2}, \diamond_{2}\right) \longmapsto\left(B_{3}, \diamond_{3}\right) \longmapsto \cdots,
$$

We will call this chain the approximating chain of $F_{\mathbf{V}}(P)$.

The crucial point of our method is that we can prove that, in a fairly general setting, it is possible to obtain the approximating chain of $F_{\mathbf{V}}(P)$ by a uniform construction, using a notion of free image-total functor on a given category $\mathbf{p} \mathbf{V}$ of partial algebras, as we describe in Section 2. The total algebras in $\mathbf{p V}$ form a full subcategory $\mathbf{V}$ of $\mathbf{p V}$. We give conditions on the functor so that repeated application of it yields the approximating chain of the free total $\mathbf{V}$ algebra over a given finite $\mathbf{p V}$ algebra. To obtain the approximating chain of the 
free $\mathbf{V}$ algebra over a given set, it then remains to describe the first $\mathbf{p V}$ algebra of the chain, which is often easy to do.

In Section 3 we show that a set of quasi-equations $\mathcal{E}$ of rank at most 1 naturally gives rise to a free image-total functor $F_{\mathcal{E}}$ on the subcategory $\mathbf{p} \mathbf{V}_{\mathcal{E}}$ of partial algebras satisfying the quasi-equations in $\mathcal{E}^{2}$

To determine, for a set of quasi-equations $\mathcal{E}$, whether $F_{\mathcal{E}}$ has the required properties for the results of Section 2 to apply, duality theory is a useful tool. Therefore we develop a Stone-type duality for partial modal algebras in Section 4.

To summarize, our main theoretical contributions to understanding the approximating chain are the following.

- Theorem 2.11. We show that the categorical content of the approximating-chain construction is captured by a free image-total functor on a category of partial algebras.

- Lemma 3.12. We show that any logic defined by a set of quasi-equations of rank at most 1 yields a free image-total functor.

- Theorem 3.15. We give a sufficient condition for the free image-total functor for a logic to yield the free total algebra in the colimit.

- Section 4. We describe a duality between partial modal algebras and q-frames, and show how quotients dually correspond to generated subframes under this duality.

The rest of the paper discusses important examples which are applications of these general results:

- In Section 5 we focus on the variety of S4 algebras. Using the developed duality theory, we will be able to give a concrete (dual) description of the functor $F_{\mathbf{S} 4}$. This description then enables us to show that it satisfies all the conditions we need for the general result to apply. We end Section 5 by showing how the recent work of Ghilardi [8] relates to our work.

- Our general construction also applies to the class of modal algebras satisfying $\mathbf{T}, \mathbf{K B}$ and $\mathbf{K 5}$ respectively. In Section $\mathbf{6}$ we briefly discuss these results.

- In [2], Kurz and Bezhanishvili constructed the free algebras for classes $\mathbf{V}_{\mathcal{E}}$ where $\mathcal{E}$ consists of pure rank 1 equations. They do so by describing a chain of Boolean algebras whose colimit is the free $\mathbf{V}_{\mathcal{E}}$ algebra. $^{3}$ Our method encompasses this construction, as we will outline in Section 7.

We conclude the paper by mentioning some future research questions in Section 8 .

\footnotetext{
${ }^{2}$ Any set of quasi-equations may be rewritten to a logically equivalent set of quasi-equations of rank at most 1 using flattening, see Remark 3.1.

${ }^{3}$ To be more precise, the colimit is only the Boolean reduct of the free algebra, but it possesses a canonical modal structure.
} 


\section{Chains of partial modal algebras}

We will introduce partial modal algebras, in our opinion the most natural setting for building the free algebra for a variety of modal algebras. There exists an extensive literature on partial algebras, see for example [4] and Chapter 2 of [10]. For our exposition here, we choose to introduce only the concepts we need, in order to make the paper self-contained. Many of the general results in this section could have been obtained from the existing literature on partial algebras, with the exception of the definition of free image-total functor and the theorem following it, which is original, as far as we know.

Definition 2.1. A partial modal algebra (pMA) is a pair $\left(B, \diamond^{B}\right)$, where $B$ is a Boolean algebra, and $\diamond^{B}: B \rightarrow B$ is a partial function which is defined on a Boolean subalgebra $\operatorname{dom}\left(\diamond^{B}\right)$ of $B$, such that $\diamond^{B} \perp=\perp$, and, for all $a, a^{\prime} \in \operatorname{dom}\left(\diamond^{B}\right), \diamond^{B}\left(a \vee a^{\prime}\right)=\diamond^{B} a \vee \diamond^{B} a^{\prime}$. A partial modal homomorphism from a pMA $\left(B, \diamond^{B}\right)$ to a pMA $\left(C, \diamond^{C}\right)$ is a Boolean algebra homomorphism $f: B \rightarrow C$ such that $f\left[\operatorname{dom}\left(\diamond^{B}\right)\right] \subseteq \operatorname{dom}\left(\diamond^{C}\right)$, and for all $a \in$ $\operatorname{dom}\left(\diamond^{B}\right), f\left(\diamond^{B} a\right)=\diamond^{C} f(a)$.

We denote the category of partial modal algebras with partial modal homomorphisms by pMA, and the full subcategory of partial modal algebras based on finite Boolean algebras by $\mathbf{p M A} \mathbf{A}_{\omega}$.

Note that the category MA of modal algebras is isomorphic to the full subcategory of pMA, consisting of those objects $\left(B, \diamond^{B}\right)$ with $\operatorname{dom}\left(\diamond^{B}\right)=B$, which we call total modal algebras.

Remark 2.2. 1. A more categorically motivated way to describe the category of partial modal algebras is that it is the category of diagrams of the form

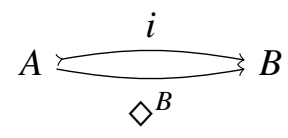

where $A$ and $B$ are Boolean algebras, $i$ is an embedding, and $\diamond^{B}: A \rightarrow B$ is a $(\perp, \vee)$ preserving function. A partial modal homomorphism from $\left(B, A, i, \diamond^{B}\right)$ to $\left(B^{\prime}, A^{\prime}, i^{\prime}, \diamond^{B^{\prime}}\right)$ can then be described as a pair of BA homomorphisms $\left(f, f^{\prime}\right)$ making the following diagrams commute:



Put into words, this simply means that partial modal algebras can also be described 
as 'Boolean algebras with a distinguished subalgebra and a modal operator from the subalgebra to the full algebra'.

2. Building on the previous remark, the invertible morphisms in the category pMA may be described as follows: a partial modal homomorphism $f:\left(B, \diamond^{B}\right) \rightarrow\left(C, \diamond^{C}\right)$ is a pMA isomorphism iff both functions $f: B \rightarrow C$ and $\left.f\right|_{\operatorname{dom}\left(\diamond^{B}\right)}: \operatorname{dom}\left(\diamond^{B}\right) \rightarrow$ $\operatorname{dom}\left(\diamond^{C}\right)$ are bijective.

3. The category pMA has two 'forgetful' functors to BA. First of all, we have the obvious $U:$ pMA $\rightarrow$ BA which sends $\left(B, \diamond^{B}\right)$ to $B$, and a pMA morphism $f$ to the same function between the underlying Boolean algebras. Secondly, we have a functor $U^{d}:$ pMA $\rightarrow$ BA which sends $\left(B, \diamond^{B}\right)$ to the Boolean algebra $\operatorname{dom}\left(\diamond^{B}\right)$, and a pMA morphism $f:\left(B, \diamond^{B}\right) \rightarrow\left(C, \diamond^{C}\right)$ to its restriction $\left.f\right|_{\operatorname{dom}\left(\diamond^{B}\right)}: \operatorname{dom}\left(\diamond^{B}\right) \rightarrow \operatorname{dom}\left(\diamond^{C}\right)$. Note that $f$ restricts correctly, by the definition of pMA morphisms. Also note that a partial modal algebra $\left(B, \diamond^{B}\right)$ is total precisely when $U\left(B, \diamond^{B}\right)=U^{d}\left(B, \diamond^{B}\right)$.

In Proposition 3.10, we will show that $U^{d}$ has a left adjoint, constructing a 'free partial modal algebra' over a given Boolean algebra.

4. One could extend the concept of partial modal algebra to more general classes of Boolean algebras with additional operators, and we will define 'partial algebras for a functor' in Section 7. We choose to focus on partial modal algebras for the larger part of this paper, since our applications in this paper lie in that field, but we notice that the material in this section is more widely applicable in other varieties which have a locally finite reduct.

As in usual universal algebra, we have an equational and quasi-equational theory of partial algebras.

Definition 2.3. Let $\left\{s_{i}, t_{i} \mid 1 \leq i \leq m\right\} \cup\{s, t\}$ be a collection of modal terms of rank at most 1 in variables $\left\{p_{1}, \ldots, p_{k}, q_{1}, \ldots, q_{l}\right\}$, such that the variables $p_{1}, \ldots, p_{k}$ are exactly the variables occurring in the scope of $\diamond$ in any of the terms $\left\{s_{i}, t_{i} \mid 1 \leq i \leq m\right\} \cup\{s, t\}$.

We say a partial modal algebra $\left(B, \diamond^{B}\right)$ satisfies the quasi-equation $\left(s_{1} \approx t_{1} \& \cdots \& s_{m} \approx\right.$ $\left.t_{m}\right) \rightarrow(s \approx t)$ iff the quasi-equation is true for all assignments of the variables $p_{i}$ by elements $a_{i} \in \operatorname{dom}\left(\diamond^{B}\right)$ and of the variables $q_{j}$ by $b_{j} \in B$.

Let $\mathcal{E}$ be a set of quasi-equations of rank at most 1 . We write $\mathbf{p} \mathbf{V}_{\mathcal{E}}$ for the full subcategory of pMA consisting of the partial modal algebras $\left(B, \diamond^{B}\right)$ which satisfy all equations in $\mathcal{E}$.

Note that we restrict ourselves to quasi-equations of rank at most 1 , as allowing terms of higher rank would require multiple applications of $\diamond$ to some of the variables, while there is no guarantee that if $a \in \operatorname{dom}(\diamond)$, then $\diamond a \in \operatorname{dom}(\diamond)$. As remarked in the Introduction, this is no real restriction, because any set of quasi-equations may be rewritten to a logically equivalent set of quasi-equations of rank at most 1 (see also Remark 3.1). 
We now state some results regarding the preservation of terms and (quasi-)equations in homomorphic images, subalgebras, and colimits of partial algebras. The proofs of these results are similar to their counterparts in universal algebra, so we will often omit them.

We say a pMA morphism $h$ is an embedding if $h$ is injective, and we say it is a quotient if it is surjective and $h\left[\operatorname{dom}\left(\diamond^{B}\right)\right]=\operatorname{dom}\left(\diamond^{C}\right)$.

Lemma 2.4 (Preservation of terms and equations of rank $\leq 1)$. Let $h:\left(B, \diamond^{B}\right) \rightarrow\left(C, \diamond^{C}\right)$ be a pMA morphism and let $s\left(p_{1}, \ldots, p_{k}, q_{1}, \ldots, q_{l}\right), t\left(p_{1}, \ldots, p_{k}, q_{1}, \ldots, q_{l}\right)$ be modal terms of rank at most 1 such that no $q_{i}$ is in the scope of $\diamond$ in $s$ or $t$. Then the following properties hold.

1. For all $a_{1}, \ldots, a_{k} \in \operatorname{dom}\left(\diamond^{B}\right), b_{1}, \ldots, b_{l} \in B$ :

$$
h\left(s^{B}\left(a_{1}, \ldots, a_{k}, b_{1}, \ldots, b_{l}\right)\right)=s^{C}\left(h\left(a_{1}\right), \ldots, h\left(a_{k}\right), h\left(b_{1}\right), \ldots, h\left(b_{l}\right)\right) .
$$

2. If $h$ is a quotient and $\left(B, \diamond^{B}\right)$ satisfies $s \approx t$, then $\left(C, \diamond^{C}\right)$ satisfies $s \approx t$.

3. If $h$ is an embedding and $\left(C, \diamond^{C}\right)$ satisfies $s \approx t$, then $\left(B, \diamond^{B}\right)$ satisfies $s \approx t$.

We will now show that we can take colimits (in algebraic terms, direct limits) of certain chains of partial modal algebras, and that the quasi-equations which hold throughout the chain still hold in the colimit. Let us first recall the definition of colimit in this setting.

Definition 2.5. Let $\left(\alpha_{n}:\left(B_{n}, \diamond_{n}\right) \rightarrow\left(B_{n+1}, \diamond_{n+1}\right)\right)_{n \in \mathbb{N}}$ be a chain of partial modal algebras with pMA morphisms between them. We say that a partial modal algebra $\left(B_{\omega}, \diamond^{B_{\omega}}\right)$, equipped with pMA morphisms $k_{n}:\left(B_{n}, \diamond_{n}\right) \rightarrow\left(B_{\omega}, \diamond^{B_{\omega}}\right)$ for every $n$, is the direct limit or colimit of this chain if, for every co-cone of pMA morphisms $\left(f_{n}:\left(B_{n}, \diamond_{n}\right) \rightarrow\left(C, \diamond^{C}\right)\right)_{n \in \mathbb{N}}$, there exists a unique pMA morphism $\bar{f}:\left(B_{\omega}, \diamond^{B_{\omega}}\right) \rightarrow\left(C, \diamond^{C}\right)$ such that $\bar{f} \circ k_{n}=f_{n}$ for all $n$.

We now specialize to a situation where we can show that the colimit exists, simply by lifting the colimit from Boolean algebras to partial modal algebras. The extra condition we need for this to work is that the maps $\alpha_{n}$ in the chain are image-total, in the sense of the following definition and theorem. In particular, we will see below that the approximating chain for the free algebra is of this form.

Definition 2.6. A pMA morphism $f:\left(B, \diamond^{B}\right) \rightarrow\left(C, \diamond^{C}\right)$ is image-total if $f[B] \subseteq \operatorname{dom}\left(\diamond^{C}\right)$.

Theorem 2.7 (Colimits of image-total chains). Let $\left(\alpha_{n}:\left(B_{n}, \diamond_{n}\right) \rightarrow\left(B_{n+1}, \diamond_{n+1}\right)\right)_{n \in \mathbb{N}}$ be a chain of partial modal algebras and image-total pMA morphisms between them.

Let $\left(k_{n}: B_{n} \rightarrow B_{\omega}\right)_{n \in \mathbb{N}}$ be the colimit of the underlying chain of Boolean algebras. Then the following hold. 
1. There exists a unique total operation $\diamond^{B_{\omega}}: B_{\omega} \rightarrow B_{\omega}$ such that each of the functions $k_{n}$ preserves $\diamond$, i.e., for all $a \in \operatorname{dom}\left(\diamond_{n}\right), k_{n}\left(\diamond_{n} a\right)=\diamond^{B_{\omega}} k_{n}(a)$.

2. If $s \approx t$ is an equation of rank at most 1 which holds in $B_{n}$ for each $n$, then $s \approx t$ holds in $B_{\omega}$.

3. If all the maps $\alpha_{n}$ are embeddings, then item (2) holds for all quasi-equations of rank at most 1 .

4. The algebra $\left(B_{\omega}, \diamond^{B_{\omega}}\right)$ is a modal algebra, and it is the pMA-colimit of the chain.

Remark 2.8. By a theorem of Manes [12], the colimit in BA is given by lifting the colimit in Set. Concretely, the underlying set of $B_{\omega}$ can be described by taking the disjoint union $\bigsqcup_{n \in \mathbb{N}} B_{n}$, and quotienting it by the equivalence relation $\sim_{B_{\omega}}$, which is defined to be the smallest equivalence relation containing all pairs $\left\langle b_{n}, \alpha_{n}\left(b_{n}\right)\right\rangle$, for $n \in \mathbb{N}, b_{n} \in B_{n}$. The Boolean algebra operations on $B$ are then well-defined, and the $n$th 'leg' of the colimiting cone, $k_{n}$, is the inclusion of $B_{n}$ into $\bigsqcup_{n \in \mathbb{N}} B_{n}$, followed by taking the class under $\sim_{B_{\omega}}$.

Proof. 1. Note that the functions $k_{n+1} \circ \diamond_{n+1} \circ \alpha_{n}: B_{n} \rightarrow B_{\omega}$ form a cone under the diagram in Set of which $\left(k_{n}: B_{n} \rightarrow B_{\omega}\right)_{n \in \mathbb{N}}$ is the colimit:

$$
k_{n+2} \circ \diamond^{n+2} \circ \alpha_{n+1} \circ \alpha_{n}=k_{n+2} \circ \alpha_{n+2} \circ \diamond_{n+1} \circ \alpha_{n}=k_{n+1} \circ \diamond_{n+1} \circ \alpha_{n},
$$

where we have used in the first equality that $\alpha_{n+1}$ is an image-total partial modal homomorphism, and in the second equality that the $k_{n}$ form a co-cone.

By the universal property of the colimit in Set, there exists a (unique) function, which we will denote by $\diamond^{B_{\omega}}$, from $B_{\omega} \rightarrow B_{\omega}$, such that, for all $n, \diamond^{B_{\omega}} \circ k_{n}=k_{n+1} \circ \diamond_{n+1} \circ \alpha_{n}$. We thus get a total operation $\diamond^{B_{\omega}}$ on $B_{\omega}$. To see that the maps $k_{n}:\left(B_{n}, \diamond_{n}\right) \rightarrow$ $\left(B_{\omega}, \diamond^{B_{\omega}}\right)$ indeed preserve $\diamond$, note that, for $b \in \operatorname{dom}\left(\diamond_{n}\right)$, we have

$$
\diamond^{B_{\omega}} k_{n} b=k_{n+1} \diamond_{n+1} \alpha_{n} b=k_{n+1} \alpha_{n} \diamond_{n} b=k_{n} \diamond_{n} b
$$

2. This follows from Lemma 2.4.1, the definition of $\left(B_{\omega}, \diamond^{B_{\omega}}\right)$, and the assumption that each $\alpha_{n}$ is image-total:

Let $b_{1}, \ldots, b_{m} \in B_{\omega}$ be an arbitrary assignment of the variables occurring in $s \approx$ $t$. Pick $n$ sufficiently large such that $\left\{b_{1}, \ldots, b_{m}\right\} \subseteq k_{n}\left[B_{n}\right]=k_{n+1}\left[\operatorname{im}\left(\alpha_{n}\right)\right]$. Pick $a_{1}, \ldots, a_{m} \in i m\left(\alpha_{n}\right) \subseteq \operatorname{dom}\left(\diamond_{n+1}\right)$ such that $b_{i}=k_{n+1}\left(a_{i}\right)$, for $i=1, \ldots, m$. Then $s^{B_{n+1}}\left(a_{1}, \ldots, a_{m}\right)=t^{B_{n+1}}\left(a_{1}, \ldots, a_{m}\right)$ by assumption, and it follows from the definition of $\diamond^{B_{\omega}}$ and Lemma 2.4.1 that $s^{B_{\omega}}\left(b_{1}, \ldots, b_{m}\right)=t^{B_{\omega}}\left(b_{1}, \ldots, b_{m}\right)$.

3. The proof is similar, using that all $k_{n}$ are then also embeddings, so that Lemma 2.4.3 applies. 
4. The fact that $\left(B_{\omega}, \diamond^{B_{\omega}}\right)$ is a modal algebra follows from part (2), and it is straightforward to check that it satisfies the universal property for the colimit, using the definition of $\diamond^{B_{\omega}}$.

We are now ready to present our new result on obtaining a free algebra by repeated application of a functor, in the setting of partial modal algebras.

Definition 2.9. Let $\mathbf{p V}$ be a full subcategory of $\mathbf{p M A}$, and write $\mathbf{V}$ for the full subcategory of $\mathbf{p V}$ whose objects are the total algebras in $\mathbf{p V}$.

Let $B_{0} \in \mathbf{p V}$. We say an object $B_{*}$ of $\mathbf{V}$, together with a pMA morphism $k_{0}: B_{0} \nrightarrow B_{*}$, is the free total $\mathbf{V}$ algebra over $B_{0}$ if, for every $C \in \mathbf{V}$, and for every pMA morphism $f_{0}: B_{0} \rightarrow C$, there exists a unique modal algebra morphism $\bar{f}: B_{*} \rightarrow C$ such that $\bar{f} \circ k_{0}=f_{0}$.

We now state conditions on a functor $F: \mathbf{p V} \rightarrow \mathbf{p V}$ so that, given a partial algebra $B_{0} \in \mathbf{p V}$, the free total $\mathbf{V}$ algebra $B_{*}$ over $B_{0}$ can be built as the colimit of a chain which is obtained by repeatedly applying the functor $F$.

First of all, $F$ must be a free image-total functor, in the sense of the following definition.

Definition 2.10. Let $\mathbf{p V}$ be a full subcategory of pMA. We say a pair $(F, \eta)$, where $F$ is a functor on $\mathbf{p V}$, and $\eta: 1_{\mathbf{p V}} \rightarrow F$ is a natural transformation, is a free image-total pair if

1. for all $B \in \mathbf{p V}, \eta_{B}$ is image-total,

2. if $h: B \rightarrow C$ is an image-total morphism in $\mathbf{p V}$, then there exists a unique pMA morphism $\bar{h}: F B \rightarrow C$ such that $\bar{h} \circ \eta_{B}=h$.

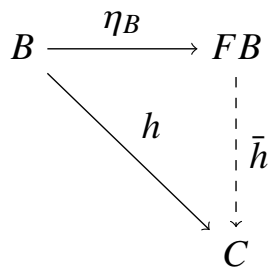

A functor $F$ on $\mathbf{p V}$ is called a free image-total functor if there exists an $\eta$ such that $(F, \eta)$ is a free image-total pair.

As mentioned in the introduction, in our intended applications, the objects of the category $\mathbf{p V}$ will form a class of partial modal algebras axiomatized by a set $\mathcal{E}$ of quasi-equations of rank at most 1, and we will be able to define a free image-total functor $F_{\mathcal{E}}$ on $\mathbf{p V}$.

Given an image-total pair $(\eta, F)$ and an object $B_{0} \in \mathbf{p} \mathbf{V}$, we now inductively define a chain in $\mathbf{p V}$ by setting, for $n \in \mathbb{N}$,

$$
B_{n+1}:=F B_{n} .
$$

This yields: 


$$
\left(B_{0}, \diamond_{0}\right) \stackrel{\eta_{B_{0}}}{\longrightarrow}\left(B_{1}, \diamond_{1}\right) \stackrel{\eta_{B_{1}}}{\longrightarrow} \cdots \stackrel{\eta_{B_{n-1}}}{\longrightarrow}\left(B_{n}, \diamond_{n}\right) \stackrel{\eta_{B_{n}}}{\longrightarrow}\left(B_{n+1}, \diamond_{n+1}\right) \stackrel{\eta_{B_{n+1}}}{\longrightarrow} \cdots
$$

in which each map $\eta_{B_{n}}$ is image-total, by assumption. We may now apply Theorem 2.7 and take the pMA-colimit $B_{\omega}$ of this chain diagram, with pMA morphisms $k_{n}: B_{n} \rightarrow B_{\omega}$ for each $n$.

In order to conclude that $B_{\omega}$ is the free total $\mathbf{V}$ algebra over $B_{0}$, we would need to show two things:

1. $B_{\omega} \in \mathbf{V}$, and

2. $B_{\omega}$ has the required universal property.

However, (1) will not be true for free image-total functors in general. In Section 3, we will state two sufficient conditions for (1) to hold (cf. Theorems 3.14 and 3.15).

The following theorem shows that it will in fact be enough to prove (1), since (2) then follows from the assumption that $F$ is a free image-total functor and general category-theoretic arguments.

Theorem 2.11. Let $\mathbf{p V}$ be a full subcategory of $\mathbf{p M A}$, let $F: \mathbf{p V} \rightarrow \mathbf{p V}$ be a free imagetotal functor, $\eta: 1 \rightarrow F$ the associated natural transformation, $B_{0} \in \mathbf{p V}$, and let $\left(k_{n}\right.$ : $\left.F^{n} B_{0} \rightarrow B_{\omega}\right)$ be the $\mathbf{p M A - c o l i m i t ~ o f ~ t h e ~ i m a g e - t o t a l ~ c h a i n ~}\left(\eta_{F^{n} B_{0}}: F^{n} B_{0} \rightarrow F^{n+1} B_{0}\right)_{n \in \mathbb{N}}$. If $B_{\omega} \in \mathbf{V}$, then $B_{\omega}$ is the free total $\mathbf{V}$ algebra over $B_{0}$.

Proof. Before proving the theorem, we first prove two lemmas.

Lemma 2.12. For any $C \in \mathbf{V}$, the map $\eta_{C}: C \rightarrow F C$ is an isomorphism.

Proof. If $C \in \mathbf{V}$, then the identity map $\operatorname{id}_{C}: C \rightarrow C$ is image-total. The result follows from applying the free image-total property of $F$ to $\mathrm{id}_{C}$.

Lemma 2.13. If $B_{\omega} \in \mathbf{V}$, then $\eta_{B_{\omega}} \circ k_{n+1}=F k_{n}$, for all $n$.

Proof. Note that $\eta_{B_{\omega}} \circ k_{n}: B_{n} \rightarrow F B_{\omega}$ is an image-total morphism, since $F B_{\omega}$ is total, being isomorphic to $B_{\omega}$ by Lemma 2.12. Now, since $\left(F k_{n}\right) \circ \eta_{B_{n}}=\eta_{B_{\omega}} \circ k_{n}$ (by naturality of $\eta$ ) and $\eta_{B_{\omega}} \circ k_{n+1} \circ \eta_{B_{n}}=\eta_{B_{\omega}} \circ k_{n}$ (as the maps $k_{n}$ form a co-cone under the chain), the result follows from the uniqueness part of the free image-total property of $F$, applied to $\eta_{B_{\omega}} \circ k_{n}$.

We now prove the theorem. Assume that $B_{\omega} \in \mathbf{V}$. Let $f_{0}: B_{0} \rightarrow C$ be a pMA morphism into some $C \in \mathbf{V}$. Inductively define $f_{n+1}: B_{n+1} \rightarrow C$ to be $\eta_{C}^{-1} \circ F f_{n}$, so that the following diagram commutes. 


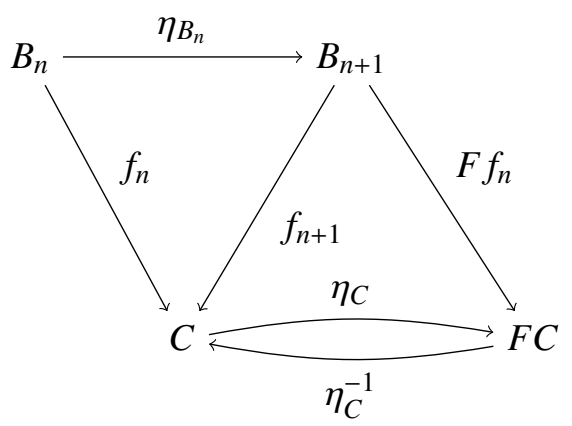

Let $\bar{f}: B_{\omega} \rightarrow C$ be the unique pMA morphism such that $\bar{f} \circ k_{n}=f_{n}$. It follows in particular that $\bar{f} \circ k_{0}=f_{0}$.

To show that $\bar{f}$ is unique, suppose that $g: B_{\omega} \rightarrow C$ is a pMA morphism such that $g \circ k_{0}=f_{0}$. It suffices to show that $g \circ k_{n}=f_{n}$, by induction. If $g \circ k_{n}=f_{n}$, then $F g \circ F k_{n}=F f_{n}$. The commutativity of the following diagram then shows that $g \circ k_{n+1}=\eta_{C}^{-1} \circ\left(F f_{n}\right)=: f_{n+1}$.

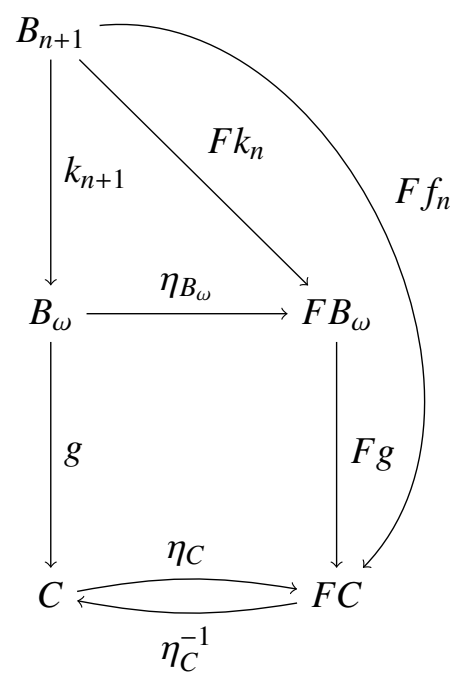

In this diagram, the upper triangle commutes by Lemma 2.13, and the lower square commutes by naturality of $\eta$.

\section{The functor for a quasi-equational class}

In this section we will show, given a set $\mathcal{E}$ of rank 0,1 quasi-equations, how to define a free image-total functor $F_{\mathcal{E}}$ on the category $\mathbf{p} \mathbf{V}_{\mathcal{E}}$ of partial modal algebras satisfying the quasi-equations in $\mathcal{E}$. Moreover, we will state sufficient conditions for the colimit of the chain arising from repeated application of the functor $F_{\mathcal{E}}$ to be in $\mathbf{p} \mathbf{V}_{\mathcal{E}}$. In the latter case, all conditions of Theorem 2.11 will be satisfied, and consequently the colimit of the chain will be the free total $\mathbf{p} \mathbf{V}_{\mathcal{E}}$ algebra over a given partial $\mathbf{p} \mathbf{V}_{\mathcal{E}}$ algebra. We end the section by showing how to then construct the free $\mathbf{V}$ algebra over a given set. 
Remark 3.1 (On rewriting arbitrary quasi-equations into quasi-equations of rank 0,1). For any set $\mathcal{E}$ of quasi-equations, there is a set $\mathcal{E}^{\prime}$ of quasi-equations of rank 0,1 such that a partial algebra satisfies $\mathcal{E}$ iff it satisfies $\mathcal{E}^{\prime}$. The method to produce this set $\mathcal{E}^{\prime}$ is completely general, and is sometimes called flattening. The idea of this method is that one may repeatedly replace higher-rank terms by newly introduced variables.

Consider for example the class of S4 algebras. This class is usually axiomatized by the equations

$$
\begin{array}{r}
a \leq \diamond a \\
\diamond \diamond a \leq \diamond a .
\end{array}
$$

The first equation is already of rank 0,1 . To rewrite the second equation, we introduce a new variable $a^{\prime}$ to replace the inner $\diamond a$ in $\diamond \diamond a$. The second equation is then equivalent to

$$
a^{\prime} \leq \diamond a \text { implies } \diamond a^{\prime} \leq \diamond a
$$

which is a rank 0,1 quasi-equation.

We will briefly sketch how this approach works in general, leaving out the details for the reader to fill in. If $\left(\bigwedge_{i=1}^{n} s_{i} \leq t_{i}\right) \rightarrow s \leq t$ is a quasi-equation in which a variable $x$ occurs with rank $>1$, say in $s$, then let $u$ be the largest subterm of $s$ in which that occurrence of $x$ is not under the scope of a diamond. Then $\diamond u$ is a subterm of $s$. Let us assume for now that $\diamond u$ occurs positively in $s$. Let $y$ be a fresh variable. Now let $s_{n+1} \leq t_{n+1}$ be the equation $y \leq \diamond u$, which is of rank 0,1 by definition. Let $s^{\prime}$ be the term $s$, except that the entire subterm $\diamond u$ is replaced by the fresh variable $y$. One may prove that an algebra satisfies the quasi-equation $\left(\bigwedge_{i=1}^{n} s_{i} \leq t_{i}\right) \rightarrow s \leq t$ iff it satisfies $\left(\bigwedge_{i=1}^{n+1} s_{i} \leq t_{i}\right) \rightarrow s^{\prime} \leq t$. The occurrence of $y$ in $s^{\prime}$ is of strictly lower rank than the occurrence of $x$ in $s$ that we started from. Now proceed by induction. $^{4}$

For the rest of this section, we fix a set $\mathcal{E}$ of rank 0,1 quasi-equations. To define the functor $F_{\mathcal{E}}: \mathbf{p} \mathbf{V}_{\mathcal{E}} \rightarrow \mathbf{p} \mathbf{V}_{\mathcal{E}}$, we will need the concept of an $\mathcal{E}$-congruence, which in turn derives from the concept of a partial modal algebra congruence. Recall that we defined a partial modal quotient to be a pMA morphism $p: B \rightarrow C$ which is surjective, and maps $\operatorname{dom}\left(\diamond^{B}\right)$ onto $\operatorname{dom}\left(\diamond^{C}\right)$.

Definition 3.2. Let $\left(B, \diamond^{B}\right)$ be a partial modal algebra. A partial modal algebra congru-

\footnotetext{
${ }^{4}$ To complete the formal proof, one would also consider the case in which $\diamond u$ occurs negatively in $s$, as well as the cases in which the occurrence of $x$ with rank $>1$ is in one of the $s_{i}, t_{i}$, or in $t$. All of these cases are treated similarly.
} 
ence on $\left(B, \diamond^{B}\right)$ is a Boolean algebra congruence $\vartheta$ on $B$ satisfying ${ }^{5}$

$$
\text { if } a, a^{\prime} \in \operatorname{dom}\left(\diamond^{B}\right) \text { and } a \approx a^{\prime} \text {, then } \diamond^{B} a \approx \diamond^{B} a^{\prime} \text {. }
$$

We now have the following connection between pMA quotients and pMA congruences, as one would expect.

Proposition 3.3. If $\vartheta$ is a pMA congruence on $\left(B, \diamond^{B}\right)$, then there exists a pMA quotient $p:\left(B, \diamond^{B}\right) \rightarrow\left(B / \vartheta, \diamond^{B / \vartheta}\right)$ such that $\operatorname{ker}(p)=\vartheta$.

Proposition 3.4. If $p:\left(B, \diamond^{B}\right) \rightarrow\left(C, \diamond^{C}\right)$ is a pMA quotient, then $\operatorname{ker}(p):=\left\{\left(b, b^{\prime}\right) \mid p(b)=\right.$ $\left.p\left(b^{\prime}\right)\right\}$ is a pMA congruence, and there exists a pMA isomorphism $f$ making the following diagram commute:

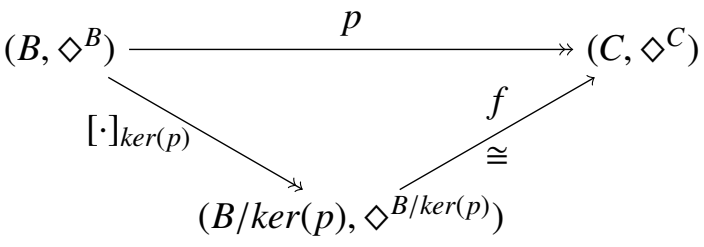

It is now natural to wonder, given a partial modal algebra $\left(B, \diamond^{B}\right)$, whether it has any congruences at all. As is to be expected, there are always two trivial pMA congruences: the diagonal $\Delta:=\{(b, b) \mid b \in B\}$ is the smallest pMA congruence on $\left(B, \diamond^{B}\right)$, and $\nabla:=B \times B$ is the largest pMA congruence on $\left(B, \diamond^{B}\right)$. The following lemma and definition show that we can define a smallest pMA congruence which identifies a given set of pairs.

Lemma 3.5. Let $\left(B, \diamond^{B}\right)$ be a partial modal algebra, and $\Theta$ a collection of pMA congruences on $B$. Then $\bigcap_{\vartheta \in \Theta} \vartheta$ is a pMA congruence on $\left(B, \diamond^{B}\right)$.

Definition 3.6. Let $\left(B, \diamond^{B}\right)$ be a partial modal algebra, and $S \subseteq B \times B$ a set of pairs. Then

$$
\Theta(S):=\bigcap\{\vartheta \subseteq B \times B \mid \vartheta \text { is a pMA congruence and } S \subseteq \vartheta\}
$$

is the smallest pMA congruence containing $S$, and we call it the pMA congruence generated by $S$.

We can now also define 'partial $\mathcal{E}$-congruences' in the obvious way, and have exactly the same theory as described above for partial MA congruences.

Definition 3.7. Let $\left(B, \diamond^{B}\right)$ be a partial modal algebra. A pMA congruence $\psi$ is called a partial $\mathcal{E}$-congruence if $\left(B / \psi, \diamond^{B / \psi}\right)$ satisfies all quasi-equations in $\mathcal{E}$.

\footnotetext{
${ }^{5}$ Given a congruence $\vartheta$, we write $a \approx_{\vartheta} a^{\prime}$ if the elements $a$ and $a^{\prime}$ are identified by the congruence $\vartheta$. We usually omit the subscript $\vartheta$ and simply write $a \approx a^{\prime}$, if it is clear from the context which congruence we mean.
} 
As before, the collection of partial $\mathcal{E}$-congruences is stable under intersections, so we get the following analogue to Definition 3.6.

Definition 3.8. Let $\left(B, \diamond^{B}\right)$ be a partial modal algebra, and $S \subseteq B \times B$ a set of pairs. Then there exists a smallest $\mathcal{E}$-congruence containing $S$, which we call the partial $\mathcal{E}$-congruence generated by $S$.

The above observation allows us to describe a left adjoint to the inclusion functor $I_{\mathcal{E}}$ : $\mathbf{p} \mathbf{V}_{\mathcal{E}} \rightarrow \mathbf{p M A}$ as follows. Let $\left(B, \diamond^{B}\right)$ be an arbitrary pMA. Denote by $\psi_{B}$ the smallest $\mathcal{E}$ congruence on $\left(B, \diamond^{B}\right)$, and let $J_{\mathcal{E}}\left(B, \diamond^{B}\right)$ be the pMA quotient of $\left(B, \diamond^{B}\right)$ by the congruence $\psi_{B}$. By definition, $J_{\mathcal{E}}\left(B, \diamond^{B}\right)$ is in $\mathbf{p} \mathbf{V}_{\mathcal{E}}$. We denote the quotient map by $\rho_{B}:\left(B, \diamond^{B}\right) \rightarrow$ $J_{\mathcal{E}}\left(B, \diamond^{B}\right)$. If $f:\left(B, \diamond^{B}\right) \rightarrow\left(C, \diamond^{C}\right)$ is a pMA morphism, then $\rho_{C} f:\left(B, \diamond^{B}\right) \rightarrow J_{\mathcal{E}}\left(C, \diamond^{C}\right)$ is a map into a $\mathbf{p} \mathbf{V}_{\mathcal{E}}$ algebra, so there is a unique factorisation $J_{\mathcal{E}} f: J_{\mathcal{E}}\left(B, \diamond^{B}\right) \rightarrow J_{\mathcal{E}}\left(C, \diamond^{C}\right)$. Thus we have constructed a functor $J_{\mathcal{E}}: \mathbf{p M A} \rightarrow \mathbf{p} \mathbf{V}_{\mathcal{E}}$.

Proposition 3.9. The functor $J_{\mathcal{E}}$ is left adjoint to the inclusion functor $I_{\mathcal{E}}: \mathbf{p} \mathbf{V}_{\mathcal{E}} \rightarrow \mathbf{p M A}$.

Proof. If $\left(C, \diamond^{C}\right) \in \mathbf{p} \mathbf{V}_{\mathcal{E}}$, then a pMA morphism $\left(B, \diamond^{B}\right) \rightarrow\left(C, \diamond^{C}\right)$ factors uniquely through $J_{\mathcal{E}}\left(B, \diamond^{B}\right)$.

We now define a left adjoint to the forgetful functor $U^{d}$ from Remark 2.2.3. We will use this left adjoint and the functor $J_{\mathcal{E}}$ to obtain a free image-total functor on $\mathbf{p} \mathbf{V}_{\mathcal{E}}$.

Intuitively, the left adjoint to $U^{d}$ acts on a Boolean algebra $B$ by formally adding elements $b$ to $B$, for all $b \in B$, and turning the resulting set into a partial modal algebra. To make this precise in Proposition 3.10 below, we recall the following free construction on the category of Boolean algebras. Let $\bullet \mathbf{B A} \rightarrow \mathbf{S L}$ be the functor from the category of Boolean algebras to the category of join-semilattices which sends a Boolean algebra $B$ to the semilattice $B:=$ $\{\diamond b \mid b \in B\}$, on which the join operation is defined by $b \vee b^{\prime}:=\diamond\left(b \vee b^{\prime}\right)$. The functor $\checkmark$ is naturally isomorphic to the forgetful functor $U: \mathbf{B A} \rightarrow \mathbf{S L}$, hence it has a left adjoint which we call $F_{\mathbf{B A}}^{\vee}$, so that $F_{\mathbf{B A}}^{\vee}(\diamond)$ is the free Boolean algebra over the join-semilattice $\checkmark B$. We use the notation $\downarrow$ for this functor to distinguish the original elements in $B$ from their counterparts in $F_{\mathbf{B A}}^{\vee}(\diamond B)$.

Proposition 3.10. Let $H: \mathbf{B A} \rightarrow$ pMA be the functor which sends a Boolean algebra $B$ to the partial modal algebra $\left(B+F_{\mathbf{B A}}^{\vee}(\bullet), \bullet\right)$, where we regard $B$ as subalgebra of $B+F_{\mathbf{B A}}^{\vee}(B)$ and let $\bullet$ be the modal operator which sends $b \in B$ to $b$ and is undefined elsewhere. For a BA homomorphism $f: B \rightarrow C$ we define $H(f): B+F_{\mathbf{B A}}^{\vee}(\diamond B) \rightarrow$ $C+F_{\mathbf{B A}}^{\vee}(\bullet)$ to be the coproduct of the assignments

$$
\begin{aligned}
b & \mapsto f(b) \quad \text { for } b \in B, \\
\diamond b & \mapsto \quad f(b) \quad \text { uniquely extended to } F_{\mathbf{B A}}^{\vee}(\diamond B) .
\end{aligned}
$$




\section{Then $H$ is left adjoint to $U^{d}$.}

Proof. Since $U^{d} H B=d o m\left(\diamond^{H B}\right) \cong B$, we have an obvious function $\zeta_{B}: B \rightarrow H B$, namely the coproduct map into the first component. We claim that the arrow $\zeta_{B}$ is universal. To see this, let $B$ be a Boolean algebra, $\left(C, \diamond^{C}\right)$ a pMA, and $f: B \rightarrow \operatorname{dom}\left(\diamond^{C}\right)$ a BA homomorphism. Define

$$
\begin{aligned}
\bar{f}: B+F_{\mathbf{B A}}^{\vee}(\diamond B) & \rightarrow C & \\
b & \mapsto f(b) & \text { for } b \in B, \\
\diamond b & \mapsto \diamond^{C} f(b) & \text { uniquely extended to } F_{\mathbf{B A}}^{\vee}(\diamond B) .
\end{aligned}
$$

Then $\bar{f} \zeta_{B}=f$, and it is clear that $\bar{f}$ is the unique pMA morphism $H B \rightarrow C$ with this property.

We are now ready to define a free image-total functor $F_{\mathcal{E}}: \mathbf{p} \mathbf{V}_{\mathcal{E}} \rightarrow \mathbf{p} \mathbf{V}_{\mathcal{E}}$. Essentially this functor sends a $\mathbf{p} \mathbf{V}_{\mathcal{E}}\left(B, \diamond^{B}\right)$ first to the partial modal algebra $H(B)=\left(B+F_{\mathbf{B A}}^{\vee}(\diamond B), \diamond\right)$, defined in Propostion 3.10, and thereafter takes the smallest $\mathbf{p} \mathbf{V}_{\mathcal{E}}$-quotient ensuring that the newly defined partial operator agrees with the old one. This is made precise as follows.

Definition 3.11. Let $\left(B, \diamond^{B}\right)$ be a partial $\mathbf{V}_{\mathcal{E}}$ algebra. We define

$$
F_{\mathcal{E}}\left(B, \diamond^{B}\right)=\left(B+F_{\mathbf{B A}}^{\vee}(\diamond B), \diamond\right) / \vartheta_{B},
$$

where $\vartheta_{B}$ denotes the smallest $\mathbf{p} \mathbf{V}_{\mathcal{E}}$ congruence on $\left(B+F_{\mathbf{B A}}^{\vee}(\diamond B), \diamond\right)$ satisfying

$$
\forall a \in \operatorname{dom}\left(\diamond^{B}\right): \diamond a \approx \diamond^{B} a
$$

To define $F$ on morphisms, let $f:\left(B, \diamond^{B}\right) \rightarrow\left(C, \diamond^{C}\right)$ be a morphism in $\mathbf{p} \mathbf{V}_{\mathcal{E}}$. First define $\tilde{f}$ to be the pMA morphism $B+F_{\mathbf{B A}}^{\vee}(\diamond B) \stackrel{H(f)}{\longrightarrow} C+F_{\mathbf{B A}}^{\vee}(\diamond C) \stackrel{[-]_{\vartheta} C}{\longrightarrow} F_{\mathcal{E}} C$. As $\vartheta_{B} \subseteq \operatorname{ker}(\tilde{f})$, $\tilde{f}$ factors uniquely through the quotient $F_{\mathcal{E}} B$, and thus yields a well-defined function $F_{\mathcal{E}} f$ : $F_{\mathcal{E}} B \rightarrow F_{\mathcal{E}} C$ which sends $[b]_{\vartheta_{B}}$ to $\tilde{f}(b)$.

Finally we define a natural transformation $\eta: 1 \rightarrow F_{\mathcal{E}}$, whose component $\eta_{B}: B \rightarrow F_{\mathcal{E}} B$ is given by $b \mapsto[b]_{\vartheta_{B}}$.

Lemma 3.12. The pair $\left(F_{\mathcal{E}}, \eta\right)$ is a free image-total pair on $\mathbf{p} \mathbf{V}_{\mathcal{E}}$.

Proof. Clearly, for all $B \in \mathbf{p V}_{\mathcal{E}}, \eta_{B}$ is image-total. Now let $h: B \rightarrow C$ be an image-total morphism in $\mathbf{p} \mathbf{V}_{\mathcal{E}}$. First define the function

$$
\begin{aligned}
& \tilde{h}: B+F_{\mathbf{B A}}^{\vee}(\diamond B) \rightarrow C \\
& b \mapsto h(b) \quad \text { for } b \in B, \\
& \diamond b \mapsto \diamond^{C} h(b) \quad \text { uniquely extended to } F_{\mathbf{B A}}^{\vee}(\diamond B) \text {. }
\end{aligned}
$$


As $h$ is image total, the function $\tilde{h}$ is well-defined. From the fact that $h$ is a morphism in $\mathbf{p} \mathbf{V}_{\mathcal{E}}$ it follows that $\vartheta_{B} \subseteq \operatorname{ker}(\tilde{h})$, hence $\tilde{h}$ factors uniquely through the quotient $F_{\mathcal{E}} B$ yielding a map $\bar{h}: F_{\mathcal{E}} B \rightarrow C$. One readily checks that $\bar{h}$ is the unique extension of $h$ satisfying $\bar{h} \circ \eta_{B}=h$.

We may now apply Theorem 2.11 to derive the following.

Proposition 3.13. Let $B_{0}$ be a partial $\mathbf{V}_{\mathcal{E}}$ algebra and let $\left(k_{n}: F_{\mathcal{E}}^{n} B_{0} \rightarrow B_{\omega}\right)$ be the pMAcolimit of the image-total chain $\left(\eta_{F_{\mathcal{E}}^{n} B_{0}}: F_{\mathcal{E}}^{n} B_{0} \rightarrow F_{\mathcal{E}}^{n+1} B_{0}\right)_{n \in \mathbb{N}}$. If $B_{\omega}$ is in $\mathbf{p} \mathbf{V}_{\mathcal{E}}$, then $B_{\omega}$ is the free total $\mathbf{V}_{\mathcal{E}}$ algebra over $B_{0}$.

Proof. Combine Theorem 2.11 and Lemma 3.12.

The following two theorems now follow from this proposition and Theorem 2.7. They state sufficient conditions for the colimit $B_{\omega}$ of the chain for $B_{0}$ (described in the above theorem) to be in $\mathbf{p} \mathbf{V}_{\mathcal{E}}$.

Theorem 3.14. If $\mathcal{E}$ is a set of equations of rank at most 1 , then $B_{\omega}$ is in $\mathbf{V}_{\mathcal{E}}$, whence it is the free total $\mathbf{V}_{\mathcal{E}}$ algebra over $B_{0}$.

Theorem 3.15. If $\mathcal{E}$ is a set of quasi-equations of rank at most 1 , and, for each $n$, the morphism $\eta_{F_{\mathcal{E}}^{n} B_{0}}$ is an embedding, then $B_{\omega}$ is in $\mathbf{V}_{\mathcal{E}}$, whence it is the free total $\mathbf{V}_{\mathcal{E}}$ algebra over $B_{0}$.

The above theorems describe situations in which the forgetful functor $U_{\mathbf{p} \mathbf{V}_{\mathcal{E}}}^{\mathbf{V}_{\mathcal{E}}}: \mathbf{V}_{\mathcal{E}} \rightarrow \mathbf{p} \mathbf{V}_{\mathcal{E}}$ has a left adjoint, which we will denote by $F_{\mathbf{p} \mathbf{V}_{\varepsilon}}^{\mathbf{V}_{\varepsilon}}$. In this setting, Propositions 3.10 and 3.9 allow us to describe the free $\mathbf{V}_{\mathcal{E}}$ algebra over a given set $P$.

Theorem 3.16. Let $\mathcal{E}$ be a set of quasi-equations of rank at most 1 such that the forgetful functor $U_{\mathbf{p} \mathbf{V}_{\mathcal{E}}}^{\mathbf{V}_{\mathcal{E}}}$ has a left adjoint. For a set $P$, the total $\mathbf{V}_{\mathcal{E}}$ algebra $F_{\mathbf{p} \mathbf{V}_{\mathcal{E}}}^{\mathbf{V}_{\mathcal{E}}}\left(J_{\mathcal{E}}\left(H\left(F_{\mathbf{B A}}(P)\right)\right)\right)$ is the free $\mathbf{V}_{\mathcal{E}}$ algebra over $P$.

Proof. Combine the universal properties of $F_{\mathbf{B A}}, H, J_{\mathcal{E}}$ and $F_{\mathbf{p} \mathbf{V}_{\mathcal{E}}}^{\mathbf{V}_{\mathcal{E}}}$.

For a set of quasi-equations $\mathcal{E}$ and a set $P$ of variables, we define a chain of $\mathbf{p} \mathbf{V}_{\mathcal{E}}$ algebras by setting $B_{0}=J_{\mathcal{E}}\left(H\left(F_{\mathbf{B A}}(P)\right)\right)$ and inductively defining, for $n \in \mathbb{N}$,

$$
B_{n+1}=F_{\mathcal{E}}\left(B_{n}\right),
$$

and letting the morphism $B_{n} \rightarrow B_{n+1}$ be $\eta_{B_{n}}$. In case the set $P$ is finite, all the algebras in this chain will be finite. In case, for each $n \in \mathbb{N}$, the map $\eta_{B_{n}}$ is an embedding, it follows from the above theorems that the colimit of this chain is the free $\mathbf{V}_{\mathcal{E}}$ algebra over $P$. The algebras in this chain then approximate the (generally infinite) free algebra by its finite pieces, allowing as a direct application, for example, a procedure to decide equivalence of $\mathbf{V}_{\mathcal{E}}$ terms. 
Note that in case $\mathcal{E}$ is axiomatized by equations, the above defined chain always yields the free $\mathbf{V}_{\mathcal{E}}$ algebra as its colimit. However, if the morphisms in the chain are not embeddings, the algebras do not give a faithful approximation of the total free algebra. Hence, it is essential to determine whether the morphisms in the chain are embeddings. Duality theory may be a useful tool in this regard. Therefore, we develop a duality theory for partial modal algebras in the next section. In Section 5 we study the particular case of S4 algebras and apply the developed duality to show that, for each finite partial S4 algebra $B$, the mapping $\eta_{B}$ is an embedding. Whence, the above results apply to $\mathbf{S 4}$. In addition, the duality will enable us to give a concrete description of the (duals of) the algebras in the chain approximating the free $\mathrm{S} 4$ algebra.

Using duality one may also show that our construction applies to the class of modal algebras satisfying T, KB and $\mathbf{K 5}$ respectively. We briefly discuss these results in Section 6.

\section{Duality for partial modal algebras}

In this section we describe a duality for finite partial modal algebras. As is to be expected, this duality is closely related to the duality for (total) modal algebras. Readers unfamiliar with this duality are referred to [3] or [9].

We will focus on finite partial modal algebras as we only encounter those in our current application, and this makes the technical details of the duality a bit easier, since topology can then be left out of the picture. However, one may show that this duality for finite algebras is the restriction to the finite case of a general Stone-type duality.

From the first item in Remark 2.2, we see that partial modal algebras dually correspond to 'Kripke frames with a distinguished quotient and a relation into the quotient'. This leads us to define the following Kripke structures for partial modal algebras, which we will call 'q-frames' because we think of them as 'Kripke frames with a quotient'.

Definition 4.1. A q-frame is a triple $(X, \sim, R)$, where $\sim$ is an equivalence relation on $X$, and $R$ is a relation on $X$ such that for all $x, y, y^{\prime} \in X$, if $x R y \sim y^{\prime}$, then $x R y^{\prime}$ (i.e., $R \circ \sim \subseteq R$ ).

A bounded morphism from a q-frame $\left(X, \sim_{X}, R_{X}\right)$ to a q-frame $\left(Y, \sim_{Y}, R_{Y}\right)$ is a function $f: X \rightarrow Y$ such that

1. if $x \sim_{X} x^{\prime}$, then $f(x) \sim_{Y} f\left(x^{\prime}\right)$,

2. if $x R_{X} x^{\prime}$, then $f(x) R_{Y} f\left(x^{\prime}\right)$,

3. if $f(x) R_{Y} y$, then there exists $x^{\prime} \in X$ such that $x R_{X} x^{\prime}$ and $f\left(x^{\prime}\right) \sim_{Y} y$.

We denote the category of q-frames with bounded morphisms by $\mathbf{q F r}$, and the full subcategory of q-frames based on a finite set by $\mathbf{q F r} \mathbf{r}_{\omega}$. 
Remark 4.2. Again, q-frames could also be equivalently described as structures of the form $\left(X, X^{\prime}, q, R\right)$, where $q: X \rightarrow X^{\prime}$ is a surjective map and $R$ is a relation from $X$ to $X$. The definition of bounded morphism can then also be reformulated with commutative diagrams, in a similar way as we did in Remark 2.2.1.

Condition 3 is a generalization of the notion of 'relativized openess', which was introduced by Ghilardi in [6]. He only works with preordered structures, but generalizing his notion to more general relations, one would formulate Condition 3 in his language by saying that the map $f$ is $q$-open.

We now have the following duality between finite partial modal algebras and finite q-frames.

Theorem 4.3. There is a dual equivalence between the categories $\mathbf{p} \mathbf{M A} \mathbf{A}_{\omega}$ and $\mathbf{q F r} \mathbf{r}_{\omega}$.

Proof. The quickest way to see that this is true is using the categorical framework outlined in Remarks 2.2.1 and 4.2 and the basic Stone duality Set $_{\omega}^{\mathrm{op}} \simeq \mathbf{B A}_{\omega}$. However, the following proof gives a more concrete description of the duality, which we will use later. We define functors $\Phi: \mathbf{q F r} \mathbf{r}_{\omega}^{\text {op }} \leftrightarrows \mathbf{p M A}: \Psi$. For a q-frame $(X, \sim, R)$, let $\Phi(X, \sim, R)$ be the partial modal algebra $\left(B, \diamond^{B}\right)$, where

- $B:=\mathcal{P}(X)$,

- $\operatorname{dom}\left(\diamond^{B}\right):=\{U \in \mathcal{P}(X) \mid U$ is $\sim$-saturated $\}$,

- for $U \in \operatorname{dom}\left(\diamond^{B}\right), \diamond^{B}(U):=\{x \in X \mid \exists y \in U: x R y\}=R^{-1}[U]$.

For a partial modal algebra $\left(B, \diamond^{B}\right)$, let $\Psi\left(B, \diamond^{B}\right)$ be the q-frame $(X, \sim, R)$, where

- $X=\mathbf{A t}(B)$, the set of atoms of $B$,

- $x \sim x^{\prime}$ iff for all $a \in \operatorname{dom}\left(\diamond^{B}\right), x \leq a \leftrightarrow x^{\prime} \leq a$,

- $x R x^{\prime}$ iff $x \leq \diamond^{B}\left(\wedge\left\{a \in \operatorname{dom}\left(\diamond^{B}\right) \mid x^{\prime} \leq a\right\}\right)$.

To understand the definition of the relation $R$, note that $\bigwedge\left\{a \in \operatorname{dom}\left(\diamond^{B}\right): x^{\prime} \leq a\right\}$ is the 'best approximation' of the element $x^{\prime} \in \mathbf{A t}(B)$ by an element of $\operatorname{dom}\left(\diamond^{B}\right)$ : it is the value of the left adjoint of the inclusion homomorphism $i: \operatorname{dom}\left(\diamond^{B}\right) \hookrightarrow B$. Unravelling the definitions and using the known duality $\operatorname{Set}_{\omega}^{\mathrm{op}} \simeq \mathbf{B A}_{\omega}$, one may now show that $\left(B, \diamond^{B}\right) \cong \Phi \Psi\left(B, \diamond^{B}\right)$, for any finite partial modal algebra $\left(B, \diamond^{B}\right)$ and $(X, \sim, R) \cong \Psi \Phi(X, \sim, R)$, for any finite qframe $(X, \sim, R)$.

Regarding morphisms, we also rely on the known duality $\mathbf{S e t}_{\omega}^{\mathrm{op}} \simeq \mathbf{B} \mathbf{A}_{\omega}$, as follows. If $f:\left(X, \sim_{X}, R_{X}\right) \rightarrow\left(Y, \sim_{Y}, R_{Y}\right)$ is a bounded morphism between q-frames, let $\Phi(f):=f^{-1}$, as in the duality Set $_{\omega}^{\mathrm{op}} \simeq \mathbf{B} \mathbf{A}_{\omega}$. This is a BA homomorphism from $\mathcal{P}(Y)$ to $\mathcal{P}(X)$, and one may check from the definitions that it is in fact a partial modal homomorphism from $\Phi\left(Y, \sim_{Y}, R_{Y}\right)$ to $\Phi\left(X, \sim_{X}, R_{X}\right)$. In the other direction, if $h:\left(B, \diamond^{B}\right) \rightarrow\left(C, \diamond^{C}\right)$ is a partial modal homomorphism, let $\Psi(h):=\left.h^{b}\right|_{\operatorname{At}(C)}$ be the function $\operatorname{At}(C) \rightarrow \operatorname{At}(B)$ which we 
get from the duality $\mathbf{S e t}_{\omega}^{\mathrm{op}} \simeq \mathbf{B} \mathbf{A}_{\omega}$. Again, one may check that $f$ is a bounded morphism from $\Psi\left(C, \diamond^{C}\right)$ to $\Psi\left(B, \diamond^{B}\right)$. We already know from the duality $\mathbf{S e t}_{\omega}^{\mathrm{op}} \simeq \mathbf{B A}_{\omega}$ that these assignments on morphisms are mutually inverse and natural, which concludes the proof.

We now develop, in the partial setting, an analogue of the correspondence between quotients of modal algebras and generated subframes of Kripke frames.

Definition 4.4. Let $\left(X, \sim_{X}, R_{X}\right)$ be a q-frame. We say $\left(Y, \sim_{Y}, R_{Y}\right)$ is a generated sub-qframe of $\left(X, \sim_{X}, R_{X}\right)$ if we have $Y \subseteq X, \sim_{Y}=\sim_{X} \cap(Y \times Y), R_{Y}=R_{X} \cap(Y \times Y)$, and

$$
\text { if } y \in Y, x \in X \text {, and } y R_{X} x \text {, then there exists } x^{\prime} \in Y \text { with } x \sim_{X} x^{\prime} .6
$$

An embedding of a q-frame $\left(Y, \sim_{Y}, R_{Y}\right)$ into a q-frame $\left(X, \sim_{X}, R_{X}\right)$ is a bounded morphism $i:\left(Y, \sim_{Y}, R_{Y}\right) \rightarrow\left(X, \sim_{X}, R_{X}\right)$ such that both $i: Y \rightarrow X$ and $\bar{i}: Y / \sim_{Y} \rightarrow X / \sim_{X}$ are injective functions. An isomorphism of q-frames is an embedding $i$ for which moreover both $i$ and $\bar{i}$ are surjective.

Lemma 4.5. Let $\left(X, \sim_{X}, R_{X}\right)$ and $\left(Y, \sim_{Y}, R_{Y}\right)$ be q-frames. The following are equivalent:

1. There exists an embedding $i:\left(Y, \sim_{Y}, R_{Y}\right) \longmapsto\left(X, \sim_{X}, R_{X}\right)$,

2. $\left(Y, \sim_{Y}, R_{Y}\right)$ is isomorphic to a generated sub-q-frame of $\left(X, \sim_{X}, R_{X}\right)$.

Now, using the duality and the characterizations of quotients of partial modal algebras and embeddings of q-frames, we can quickly deduce the following correspondence.

Proposition 4.6. Let $\left(B, \diamond^{B}\right)$ be a finite partial modal algebra and $\left(X, \sim_{X}, R_{X}\right)$ its dual $q$ frame. There is a one-to-one correspondence between pMA congruences on $\left(B, \diamond^{B}\right)$ and generated sub-q-frames of $\left(X, \sim_{X}, R_{X}\right)$.

Proof. By Proposition 3.4, pMA congruences on $\left(B, \diamond^{B}\right)$ correspond to isomorphism classes of pMA quotients of $\left(B, \diamond^{B}\right)$, which correspond to isomorphism classes of embeddings into the dual q-frame $\left(X, \sim_{X}, R_{X}\right)$ by the duality (Theorem 4.3), which correspond to generated sub-q-frames of $\left(X, \sim_{X}, R_{X}\right)$ by Lemma 4.5 .

Suppose a pMA congruence is generated by a given set of pairs, in the sense of Definition 3.6. We can now calculate the generated sub-q-frame that the quotient corresponds to, as follows.

Proposition 4.7. In the context of the previous proposition, if $S \subseteq B \times B$ is a set of pairs, then the pMA congruence $\Theta(S)$ generated by $S$ corresponds to the largest generated sub-q-frame of $(X, \sim, R)$ whose domain is a subset of the set

$$
P(S):=\left\{x \in X \mid \forall\left(b, b^{\prime}\right) \in S: x \leq b \leftrightarrow x \leq b^{\prime}\right\} .
$$

\footnotetext{
${ }^{6}$ Note that, in this situation, it also follows that $y R_{Y} x^{\prime}$, since $R_{X} \circ \sim_{X} \subseteq R_{X}$.
} 
In the following section we will use this duality to construct the free $\mathrm{S} 4$ algebra over a finite set of variables.

\section{Partial S4 algebras}

In Remark 3.1, we have shown how the usual equations for the class of S4 modal algebras may be rewritten into equivalent quasi-equations of rank 0,1 . This axiomatization leads to the following definition.

Definition 5.1. A partial $\mathbf{S 4}$ algebra is a partial modal algebra satisfying the quasi-equations

1. $a \leq \diamond a$,

2. $a \leq \diamond a^{\prime}$ implies $\diamond a \leq \diamond a^{\prime}$.

We write pS4 for the class of partial S4 algebras, and $\mathbf{p S 4} \mathbf{H}_{\omega}$ for the full subcategory of finite partial S4 algebras.

In this section we will give a dual description of the functor $F_{\mathbf{S 4}}$ (restricted to $\mathbf{p S 4} \mathbf{4}_{\omega}$ ) and the natural transformation $\eta$. This will enable us to show that, for each finite $\mathbf{p S 4}$ algebra $B, \eta_{B}$ is injective. Hence, the construction described in Section 3 may be applied to build, for a finite set $P$, a chain of embeddings of finite pS4 algebras whose colimit is the free S4 algebra over $P$. Moreover, the dual description of $F_{\mathbf{S} 4}$ will give a concrete description of the duals of the algebras in this chain.

\subsection{Duality for partial S4 algebras}

We start by describing which q-frames correspond to partial S4 algebras. Since we know that $\mathrm{S} 4$ algebras correspond to qosets, i.e., Kripke frames whose relations are quasiorders (reflexive and transitive), it is reasonable to suspect that something similar happens for qframes. This is why we choose to call the frames corresponding to partial S4 algebras "q-qosets".

Definition 5.2. We say a q-frame $(X, \sim, R)$ is a q-qoset if

1. $R$ is reflexive,

2. for all $x, y \in X$, if $x R y$, then there exists $y^{\prime} \sim y$ such that $R\left[y^{\prime}\right] \subseteq R[x]$.

We denote the full subcategory of $\mathbf{q F r}$ whose objects are q-qosets by qQoset.

Let $\left(X, \sim_{X}, R_{X}\right)$ be a q-frame. We say $\left(Q, \sim_{Q}, R_{Q}\right)$ is a generated sub-q-qoset of $\left(X, \sim_{X}, R_{X}\right)$ if it is a generated sub-q-frame of $\left(X, \sim_{X}, R_{X}\right)$, which is moreover a q-qoset. 
Remark 5.3. Intuitively, the second condition in the definition of q-qoset says that $R$ is 'transitive up to $\sim$-equivalence'. ${ }^{7}$ We will see in Proposition 5.6 that this condition is the right generalization of transitivity to the setting of q-frames.

We can write the definition of a q-qoset more concisely by defining, for $x \in X$,

$$
T_{x}:=\{z \in X \mid R[z] \subseteq R[x]\}
$$

The second condition then says that $R[x] \subseteq\left[T_{x}\right]_{\sim}$, where $\left[T_{x}\right]_{\sim}$ denotes the $\sim$-saturation of the set $T_{x}$. It is not hard to see that reflexivity of $R$ is equivalent to $\left[T_{x}\right]_{\sim} \subseteq R[x]$, for all $x \in X$.

From this remark, we conclude

Lemma 5.4. A q-frame $(X, \sim, R)$ is a q-qoset iff for all $x \in X, R[x]=\left[T_{x}\right]_{\sim}$.

Partial S4 congruences on a partial modal algebra $\left(B, \diamond^{B}\right)$ with dual q-frame $(X, \sim, R)$ correspond to generated sub-q-frames of $(X, \sim, R)$, which are in addition q-qosets. The following lemma will be of use in the description of the dual of $F_{\mathbf{S} 4}$, where we have to compute the generated sub-q-qoset corresponding to a given partial S4 quotient.

Lemma 5.5. Let $\left(X, \sim_{X}, R_{X}\right)$ be a q-frame, $Q \subseteq X$ a subset, $\sim_{Q}:=\sim_{X} \cap(Q \times Q)$, and $R_{Q}:=R_{X} \cap(Q \times Q)$. Then the following are equivalent:

1. $\left(Q, \sim_{Q}, R_{Q}\right)$ is a generated sub-q-qoset of $\left(X, \sim_{X}, R_{X}\right)$.

2. $\forall q \in Q, x \in X\left(q R_{X} x \Leftrightarrow \exists x^{\prime} \in Q: x \sim x^{\prime}\right.$ and $\left.R_{X}\left[x^{\prime}\right] \subseteq R_{X}[q]\right)$.

Proof. It is not hard to see that (2) implies (1). Suppose (1) holds, and let $q \in Q$ and $x \in X$ with $q R_{X} x$. As $Q$ is the underlying set of a generated sub-q-frame, pick $p \in Q$ with $x \sim p$, which implies $q R_{Q} p$. Since $Q$ is a q-qoset, pick $x^{\prime} \in Q$ with $p \sim x^{\prime}$ and $R_{Q}\left[x^{\prime}\right] \subseteq R_{Q}[q]$. By transitivity of $\sim$, we have $x \sim x^{\prime}$, and because $R_{X} \circ \sim=R_{X}$ and $Q$ is a generated subq-frame, we also get $R_{X}\left[x^{\prime}\right] \subseteq R_{X}[q]$. For the other direction, use that $R_{X}$ is reflexive and $R_{X} \circ \sim_{X}=R_{X}$.

The justification for the definition of q-qoset lies in the following proposition.

Proposition 5.6. Let $\left(B, \diamond^{B}\right)$ be a finite partial modal algebra and $(X, \sim, R)$ its dual q-frame.

The following are equivalent:

1. $\left(B, \diamond^{B}\right)$ is a partial S4 algebra,

2. $(X, \sim, R)$ is a q-qoset.

\footnotetext{
${ }^{7}$ Note that a relation $R$ is transitive if, for all $x, y \in X$, if $x R y$, then $R[y] \subseteq R[x]$.
} 
Proof. In fact, we can show that the conditions (1) and (2) in the definitions of partial $\mathrm{S} 4$ algebra and q-qoset are equivalent, respectively. This is an exercise in correspondence theory. Regarding condition (1):

$$
\begin{aligned}
\forall a \in \operatorname{dom}\left(\diamond^{B}\right): a \leq \diamond^{B} a & \Longleftrightarrow \forall x \in \operatorname{At}\left(\operatorname{dom}\left(\diamond^{B}\right)\right): x \leq \diamond^{B} x \\
& \Longleftrightarrow \forall x \in X:[x]_{\sim} \subseteq R^{-1}[x] \\
& \Longleftrightarrow x R x
\end{aligned}
$$

For the last backward implication, one uses that $R \circ \sim=R$.

The calculation of the correspondent of condition (2) is slightly more complicated, but follows standard Sahlqvist procedures, as illustrated below. First of all, unravelling the definitions, and using $R \circ \sim=R$, we get:

$$
\begin{aligned}
\forall a, a^{\prime} \in \operatorname{dom}\left(\diamond^{B}\right)\left(a \leq \diamond^{B} a^{\prime} \rightarrow\right. & \left.\diamond^{B} a \leq \diamond^{B} a^{\prime}\right) \Longleftrightarrow \\
& \forall y \in X \forall S \subseteq X\left([y] \sim \subseteq R^{-1}[S] \rightarrow R^{-1}[y] \subseteq R^{-1}[S]\right) .
\end{aligned}
$$

Taking the contrapositive of the last condition and pulling out an existential quantifier, we see it is equivalent to

$$
\forall x, y \in X \forall S \subseteq X[x R y \wedge(\forall s \in S: \neg x R s)] \rightarrow\left[\exists y^{\prime} \in X: y^{\prime} \sim y \wedge\left(\forall s \in S: \neg y^{\prime} R s\right)\right] .
$$

Let us abbreviate the long implication after the three initial universal quantifiers by $\varphi(x, y, S)$. Note that if $\varphi\left(x, y, S_{0}\right)$ holds for some $x, y \in X$ and $S_{0} \subseteq X$, then for any $S \subseteq S_{0}$, we have that $\varphi(x, y, S)$ still holds.

The largest subset $S_{0}$ for which the antecedent is true is $S_{0}:=R[x]^{c}$, showing that this condition is in fact equivalent to the first-order condition

$$
\forall x, y \in X\left[x R y \rightarrow \exists y^{\prime} \in X: y^{\prime} \sim y \wedge\left(\forall w \in R[x]^{c}: \neg y^{\prime} R w\right)\right]
$$

which is clearly equivalent to condition (2) in the definition of q-qoset.

From this fact, we now deduce the following corollaries from Theorem 4.3, Proposition 4.6 and Proposition 4.7, respectively.

Corollary 5.7. The dual equivalence of Theorem 4.3 restricts to a dual equivalence between the categories $\mathbf{p S 4} \mathbf{}_{\omega}$ and $\mathbf{q Q o s e t}{ }_{\omega}$.

Corollary 5.8. Let $\left(B, \diamond^{B}\right)$ be a finite partial modal algebra and $\left(X, \sim_{X}, R_{X}\right)$ its dual $q$ frame. There is a one-to-one correspondence between $p S 4$ congruences on $\left(B, \diamond^{B}\right)$ and generated sub-q-qosets of $\left(X, \sim_{X}, R_{X}\right)$. 
Corollary 5.9. In the context of the previous proposition, if $S \subseteq B \times B$ is a set of pairs, then the pS4 congruence generated by $S$ corresponds to the largest generated sub-q-qoset of $(X, \sim, R)$ whose domain is a subset of the set

$$
P(S):=\left\{x \in X \mid \forall\left(b, b^{\prime}\right) \in S: x \leq b \leftrightarrow x \leq b^{\prime}\right\}
$$

\subsection{Construction of the free $\mathrm{S} 4$ algebra}

Using the duality for partial S4 algebras, we will now show that the map $\eta_{B}$ for the free image-total functor $F_{\mathbf{S 4}}$ is injective, for all finite $\mathbf{p S 4}$ algebras $B$ (Corollary 5.15). This will enable us to apply Theorem 3.15 and conclude that the colimit of the chain of algebras obtained from repeatedly applying $F_{\mathbf{S} 4}$ is the free $\mathrm{S} 4$ algebra.

To obtain the result that each $\eta_{B}$ is injective, we give a dual description of the functor $F_{\mathbf{S} 4}$. Throughout this section, $B$ will be a finite $\mathbf{p S 4}$ algebra, with dual frame $(X, \sim, R)$. We start by describing the dual of the partial modal algebra $\left(B+F_{\mathbf{B A}}^{\vee}(\diamond B), \diamond\right)$.

Lemma 5.10. The q-frame dual to $\left(B+F_{\mathbf{B A}}^{\vee}(B), \diamond\right)$ is $\left(X \times \mathcal{P}(X),=_{1}, R_{\ni}\right)$, where, for $(x, T),(y, S) \in X \times \mathcal{P}(X),(x, T)=_{1}(y, S)$ iff $x=y$ and $(x, T) R_{\ni}(y, S)$ iff $y \in T$.

Proof. It is well-known that $\operatorname{At}\left(F_{\mathbf{B A}}^{\vee}(\diamond B)\right) \cong \mathcal{P}(X)$ (for more details, see for example [14, Section 15]). Hence, as the duality turns coproducts into products, $\operatorname{At}\left(B+F_{\mathbf{B A}}^{\vee}(\diamond B)\right) \cong$ $X \times \mathcal{P}(X)$. Using the description of the dual q-frame given in the proof of Theorem 4.3 , the descriptions of the dual relations follow from a straightforward computation.

To compute the dual of $\left(B+F_{\mathbf{B A}}^{\vee}(\diamond), \diamond\right) / \vartheta_{B}$, we wish to calculate the subset $P_{X}$ of atoms of $X \times \mathcal{P}(X)$ satisfying the equality

$$
\forall a \in \operatorname{dom}\left(\diamond^{B}\right): \diamond a \approx \diamond^{B} a,
$$

which we used to define the partial S4 congruence $\vartheta_{B}$, as in Definition 3.11. By Corollary 5.9, the q-frame dual to $F_{\mathbf{S} 4} B$ is then the largest generated sub-q-qoset of $(X \times \mathcal{P}(X),=1$ , $R_{\ni}$ ) whose domain is contained in $P_{X}$.

Let $(x, T) \in X \times \mathcal{P}(X)$. We want to find conditions on $(x, T)$ so that

$$
\forall a \in \operatorname{dom}\left(\diamond^{B}\right):(x, T) \leq \diamond a \Longleftrightarrow(x, T) \leq \diamond^{B} a .
$$

The domain of $\diamond^{B}$ consists of the $\sim$-saturated subsets of $X$. As both $\diamond$ and $\diamond^{B}$ preserve joins, it suffices to consider the atoms of $\operatorname{dom}\left(\diamond^{B}\right)$, i.e., the elements of $B=\mathcal{P}(X)$ of the form $[y]_{\sim}$, where $y \in X$. As $R \circ \sim=R, R^{-1}\left[[y]_{\sim}\right]=R^{-1}[y]$. Note that

$$
(x, T) \leq \diamond^{B}[y]_{\sim} \quad \Leftrightarrow \quad x \leq R^{-1}[y] \quad \Leftrightarrow \quad y \in R[x] .
$$


Furthermore,

$$
(x, T) \leq \bullet[y]_{\sim} \quad \Leftrightarrow \quad T \cap[y]_{\sim} \neq \emptyset \quad \Leftrightarrow \quad y \in[T]_{\sim} .
$$

Hence, $(x, T)$ satisfies the equality for all $a \in \operatorname{dom}\left(\diamond^{B}\right)$ iff

$$
R[x]=[T]_{\sim} .
$$

We conclude

Lemma 5.11. The collection $P_{X}$ of atoms in $X \times \mathcal{P}(X)$ which satisfy (3) is

$$
P_{X}=\left\{(x, T) \in X \times \mathcal{P}(X) \mid R[x]=[T]_{\sim}\right\} .
$$

Therefore, the dual of the functor $F_{\mathbf{S} 4}$ takes a q-frame $(X, \sim, R)$ to the largest generated subq-qoset of $\left(X \times \mathcal{P}(X),=_{1}, R_{\ni}\right)$ whose domain is contained in $P_{X}$. Let us call the domain of this q-qoset $Q_{X}$. Applying Lemma 5.5 and filling in the definitions of the relations $=_{1}$ and $R_{\ni}$ yields that $Q_{X}$ is the largest subset $Q$ of $P_{X}$ satisfying,

$$
\forall(x, T) \in Q, y \in X:[y \in T \Leftrightarrow \exists S \subseteq X .(y, S) \in Q \text { and } S \subseteq T]
$$

Although this gives some description of the dual of the functor $F_{\mathbf{S} 4}$, we can give a more explicit description of the subset $Q_{X}$ here. Recall that we defined in Remark 5.3, for $x \in X$,

$$
T_{x}=\{z \in X \mid R[z] \subseteq R[x]\} .
$$

We noted in Lemma 5.4 that if $(X, \sim, R)$ is a q-qoset, then $\left[T_{x}\right]_{\sim}=R[x]$, i.e., $\left(x, T_{x}\right) \in P_{X}$. Now, from the fact that $Q_{X}$ satisfies (4), we deduce the following properties.

Lemma 5.12. For any element $(x, T) \in Q_{X}$,

1. $x \in T$,

2. If $y \in T$, then there exists $S \subseteq T$ such that $(y, S) \in Q_{X}$,

3. $T \subseteq T_{x}$.

Let us therefore define the auxiliary set

$$
P_{X}^{\prime}:=\left\{(x, T) \in P_{X} \mid x \in T, T \subseteq T_{x}\right\},
$$

which will contain $Q_{X}$. We are now ready to give a characterisation of the set $Q_{X}$.

Proposition 5.13. Let $(X, \sim, R)$ be a q-qoset. Let

$$
Q:=\left\{(x, T) \in P_{X}^{\prime} \mid \forall y \in T \exists S \subseteq T:(y, S) \in P_{X}^{\prime}\right\} .
$$


Then $Q$ is equal to $Q_{X}$, i.e., $Q$ is the largest generated sub-q-qoset of $(X, \sim, R)$ whose domain is contained in $P_{X}$.

Proof. By Lemma 5.12, any element $(x, T) \in Q_{X}$ will satisfy the conditions defining $Q$, so $Q_{X} \subseteq Q$. It remains to show that $Q$ indeed satisfies (4).

Suppose $(x, T) \in Q, y \in X$. First of all, if $(y, S) \in Q$ for some $S \subseteq T$, then $y \in S$, so $y \in T$.

Conversely, suppose $y \in T$. By definition of $Q$, there exists $S \subseteq T$ such that $(y, S) \in P_{X}^{\prime}$. We now aim to show that $\left(y, T \cap T_{y}\right) \in Q$. To see that $\left(y, T \cap T_{y}\right) \in P_{X}$, note that

$$
S \subseteq T \cap T_{y} \subseteq T_{y},
$$

and $[S]_{\sim}=R[y]=\left[T_{y}\right]_{\sim}$, so $\left[T \cap T_{y}\right]_{\sim}=R[y]$. As $y \in T$ (by assumption) and also $y \in T_{y}$, we have $y \in T \cap T_{y} \subseteq T_{y}$ and therefore $\left(y, T \cap T_{y}\right) \in P_{X}^{\prime}$.

To show $\left(y, T \cap T_{y}\right) \in Q$, let $z \in T \cap T_{y}$ be arbitrary. We have to find $U \subseteq T \cap T_{y}$ such that $(z, U) \in P_{X}^{\prime}$. As $z \in T \cap T_{y} \subseteq T$ and $(x, T) \in Q$, there exists $U \subseteq T$ such that $(z, U) \in P_{X}^{\prime}$. It remains to show that $U \subseteq T_{y}$, from which we then conclude $U \subseteq T \cap T_{y}$, as required. Since $z \in T_{y}$, we have $R[z] \subseteq R[y]$, which implies $T_{z} \subseteq T_{y}$. Now, since $(z, U) \in P_{X}^{\prime}$, it follows that $U \subseteq T_{z} \subseteq T_{y}$.

Note that it follows from the proof above that, for $(x, T) \in P_{X}^{\prime}$ and $y \in T$, there exists some $S \subseteq T$ with $(y, S) \in P_{X}^{\prime}$ iff $\left(y, T \cap T_{y}\right) \in P_{X}^{\prime}$. Furthermore, under these condititions, $y \in T \cap T_{y} \subseteq T_{y}$. Hence, $\left(y, T \cap T_{y}\right) \in P_{X}^{\prime}$ iff $R[y]=\left[T \cap T_{y}\right]_{\sim}$. Therefore the functor $F_{\mathbf{S} 4}$ on $\mathbf{p S 4}_{\omega}$ (as defined in the previous section) may be described dually (on objects) by

$$
\begin{aligned}
G_{\mathbf{S} 4}:(X, \sim, R) \mapsto \quad & \left(Q_{X},={ }_{1} \cap\left(Q_{X} \times Q_{X}\right), R_{\ni} \cap\left(Q_{X} \times Q_{X}\right)\right) \\
\text { where } Q_{X}= & \left\{(x, T) \in X \times \mathcal{P}(X) \mid R[x]=[T]_{\sim}, x \in T, T \subseteq T_{x}\right. \\
& \text { and } \left.\forall y \in T . R[y]=\left[T \cap T_{y}\right]_{\sim}\right\} .
\end{aligned}
$$

This mapping extends to a functor $G_{\mathbf{S 4}}$ on the category $\mathbf{q Q o s e t} \mathbf{t}_{\omega}$. More precisely, if we write $\Psi: \mathbf{p S 4}_{\omega}^{\text {op }} \rightarrow$ qQoset $_{\omega}$ for the functor in the duality from Corollary 5.7, then we showed $\left(\Psi \circ F_{\mathbf{S 4}}\right)\left(B, \diamond^{B}\right)=\left(G_{\mathbf{S 4}} \circ \Psi\right)\left(B, \diamond^{B}\right)$, for every finite partial S4 algebra $\left(B, \diamond^{B}\right)$.

The natural transformation $\eta: 1 \rightarrow F_{\mathbf{S} 4}$ corresponds dually to a natural transformation $\pi: G_{\mathbf{S} 4} \rightarrow 1$. For a finite q-qoset $X, \pi_{X}$ is the restriction of the projection function $X \times$ $\mathcal{P}(X) \rightarrow X$ to a function $Q_{X} \rightarrow X$. The following lemma shows that $\pi_{X}$ is surjective for every finite q-qoset $X$. By duality we may then conclude that $\eta_{B}$ is an embedding for every finite S4 algebra $B$.

Lemma 5.14. Let $(X, \sim, R)$ be a finite q-qoset. For all $x \in X,\left(x, T_{x}\right) \in Q_{X}$.

Proof. Let $x \in X$. We have seen that $\left(x, T_{x}\right) \in P_{X}$, and it is therefore clear that $\left(x, T_{x}\right) \in P_{X}^{\prime}$. Let $y \in T_{x}$, that is, $y \in X$ with $R[y] \subseteq R[x]$. Then $T_{y} \subseteq T_{x}$ and $\left(y, T_{y}\right) \in P_{X}^{\prime}$. Hence $\left(x, T_{x}\right) \in Q=Q_{X}$. 
Corollary 5.15. Let $B$ be a finite partial $S 4$ algebra. The partial modal homomorphism $\eta_{B}: B \rightarrow F_{\mathbf{S} 4} B$ is an embedding.

It follows that the general construction of Section 3 applies to S4, i.e., defining, for a set $P$,

$$
B_{0}=J_{\mathbf{S 4}}\left(H\left(F_{\mathbf{B A}}(P)\right)\right)=\left(B+F_{\mathbf{B A}}^{\vee}(\diamond B), \diamond\right) / \vartheta_{\mathbf{S} \mathbf{4}},
$$

where $\vartheta_{\mathbf{S} 4}$ denotes the smallest $\mathbf{p} \mathbf{V}_{\mathbf{S 4}}$ congruence on $\left(B+F_{\mathbf{B A}}^{\vee}(\diamond B), \diamond\right)$, and inductively defining, for $n \in \mathbb{N}$,

$$
B_{n+1}=F_{\mathbf{S 4}}\left(B_{n}\right)
$$

yields a chain of partial $S 4$ algebras whose colimit is the free (total) S4 algebra over $P$.

The duality allows us to give a concrete description of the algebras in this chain. The dual of $B_{0}$ is the largest generated sub-q-qoset of $\left(X_{P} \times \mathcal{P}\left(X_{P}\right),={ }_{1}, R_{\ni}\right)$, where $X_{P}=\operatorname{At}\left(F_{\mathbf{B A}}(P)\right) \cong$ $\mathcal{P}(P)$. The duals of the further partial S4 algebras in the chain may be obtained by repeatedly applying the functor $G_{\mathbf{S} 4}$ described above.

The first two q-frames in the dual chain for the 1-generated free $S 4$ algebra, i.e., $P=\{p\}$, are depicted below,
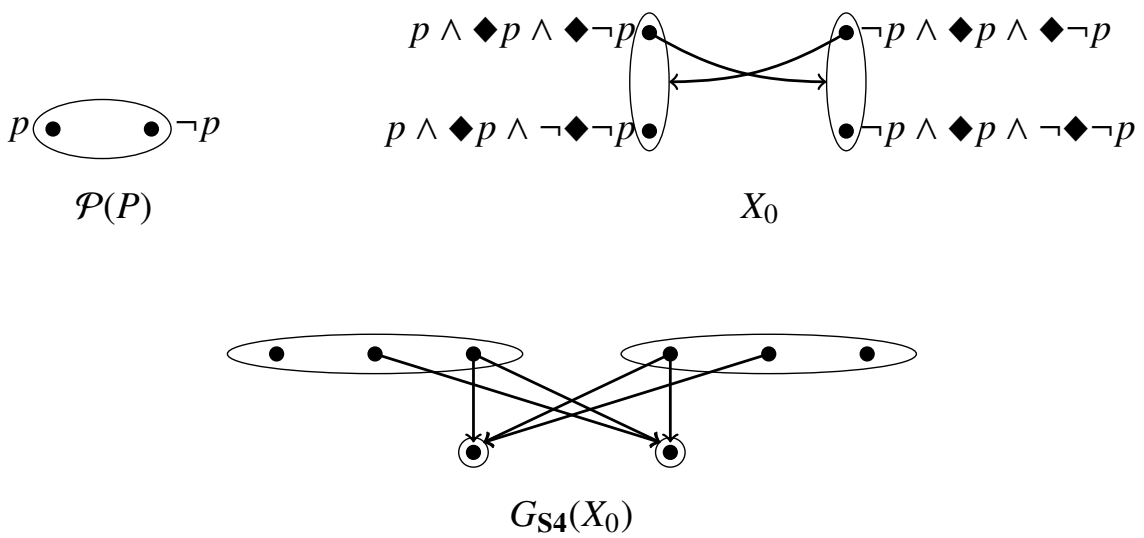

In these figures, the equivalence relation is depicted as a partition. The arrows represent the non-reflexive part of the relation $R$. Note that $R$ can indeed be regarded as a relation from points to equivalence classes of points, since $R \circ \sim=R$. Moreover, in the first two figures, the points (which are atoms of the algebra) are labelled by the formula they represent. The formulas become considerably longer in the third step, so we have omitted them.

\subsection{Comparison with the work of Ghilardi}

As stated in the introduction, our method for constructing free algebras was partly inspired by the work of Ghilardi. In this section, we will explain how the two methods relate, thereby also shedding new light on Ghilardi's construction. 
We fix a finite set $P$. In [8], Ghilardi defines a chain of total S4 algebras, with so-called continuous maps ${ }^{8}$ between them:

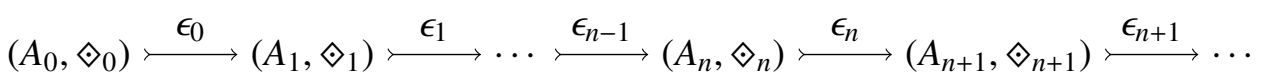

He then takes the colimit $A_{\omega}$ of the chain of underlying Boolean algebras and equips it with a modal operator $\diamond$ by defining $\diamond\left[a \in A_{n}\right]=\left[\diamond_{n+1}\left(\epsilon_{n}(a)\right) \in A_{n+1}\right]$. The modal algebra $\left(A_{\omega}, \diamond\right)$ is the free $\mathrm{S} 4$ algebra $F_{\mathbf{V}_{\mathbf{S} 4}}(P)$ over $P$. We compare this incremental construction to our chain

$$
\left(B_{0}, \diamond_{0}\right) \stackrel{\eta_{B_{0}}}{\longleftrightarrow}\left(B_{1}, \diamond_{1}\right) \stackrel{\eta_{B_{1}}}{\longleftrightarrow} \ldots \stackrel{\eta_{B_{n-1}}}{\longmapsto}\left(B_{n}, \diamond_{n}\right) \stackrel{\eta_{B_{n}}}{\longleftrightarrow}\left(B_{n+1}, \diamond_{n+1}\right) \stackrel{\eta_{B_{n+1}}}{\longmapsto} \ldots
$$

of partial S4 algebras approximating $F_{\mathbf{V}_{\mathrm{S} 4}}(P)$, as described at the end of the previous section. The underlying Boolean parts of the two chains coincide, with the only exception that our chain starts one step later, that is, $B_{n}=A_{n+1}$ and $\eta_{B_{n}}=\epsilon_{n+1}$.

The essential observation leading to our chain of partial algebras is the fact that on the image of $\epsilon_{n}$, the total operator $\diamond_{n+1}$ takes the same value as $\diamond$ does in the colimit. This image of $\epsilon_{n}$ is exactly the domain of the partial diamond in our chain. Ghilardi constructs his chain in such a way that the map $\epsilon_{n+1}$ is $\epsilon_{n}$-open, i.e., for all $a \in A_{n}, \epsilon_{n+1}\left(\diamond_{n+1} \epsilon_{n}(a)\right)=$ $\diamond_{n+2} \epsilon_{n+1}\left(\epsilon_{n}(a)\right)$. This corresponds to the fact that our map $\eta_{B_{n}}$ preserves the partial diamond. Conversely, Ghilardi's chain may be obtained from our chain of partial algebras by defining the total operator $\diamond_{n+1}$ by

$$
\diamond_{n+1}=\eta_{B_{n}}^{\mathrm{b}} \circ \diamond^{n+1} \circ \eta_{B_{n}}
$$

where $\eta_{B_{n}}^{b}$ is the left adjoint to the embedding $\eta_{B_{n}}: B_{n} \longmapsto B_{n+1}$.

Lemma 4.2 in [8] is the essential ingredient needed to prove that the colimit of Ghilardi's chain is indeed the free S4 algebra. The notion of free image-total functor, which we introduced in Definition 2.10, is already implicit in this lemma. However, Ghilardi's approach, using continuous morphisms, is tailored to work in the specific case of the logic S4. Working in the setting of partial modal algebras has enabled us to put his construction in a broader perspective.

\section{Examples}

In the previous section we have worked out in detail that our general construction applies to the class of S4 modal algebras. We now briefly discuss some other classes of modal algebras. We only state the results and leave the computations to the reader.

\footnotetext{
${ }^{8} \mathrm{~A}$ continuous map between modal algebras $\left(B, \diamond^{B}\right)$ and $\left(C, \diamond^{C}\right)$ is a Boolean algebra homomorphism $f: B \rightarrow$ $C$ satisfying in addition $\diamond^{C} f(b) \leq f\left(\diamond^{B} b\right)$, for all $b \in B$.
} 
For a finite set $P$, we write $X_{P}=\operatorname{At}\left(F_{\mathbf{B A}}(P)\right)$. First, we consider the class of $\mathrm{T}$ algebras. This is the class of modal algebras satisfying the axiom

$$
a \leq \diamond a
$$

The $\mathrm{q}$-frames corresponding to partial $\mathrm{T}$ algebras are $\mathrm{q}$-frames $(X, \sim, R)$ where the relation $R$ is reflexive. The dual of the functor $F_{\mathbf{T}}$ may be described as

$$
G_{\mathbf{T}}(X, \sim, R)=\left(\left\{(x, T) \in X \times \mathcal{P}(X) \mid R[x]=[T]_{\sim}, x \in T\right\},=_{1}, R_{\ni}\right),
$$

where we just write $={ }_{1}$ and $R_{\ni}$ for the restriction of these relations on $X \times \mathcal{P}(X)$ to the underlying set of the described $\mathrm{q}$-frame. For any $\mathrm{T} \mathrm{q}$-frame, the projection map $G_{\mathbf{T}}(X, \sim$ $, R) \rightarrow(X, \sim, R)$ is surjective. Hence, our general construction applies to the class of $\mathrm{T}$ algebras and the approximating chain of the free $\mathrm{T}$ algebra over a set $P$ may be described dually by repeated application of the functor $G_{\mathbf{T}}$ to

$$
X_{0}=\left(\left\{(x, T) \in X_{P} \times \mathcal{P}\left(X_{P}\right) \mid x \in T\right\},={ }_{1}, R_{\ni}\right),
$$

the largest generated subframe of $\left(X_{P} \times \mathcal{P}\left(X_{P}\right),=_{1}, R_{\ni}\right)$ which is a T q-frame. See [5] for more details.

Our method also applies to the class of KB algebras, i.e., the class of modal algebras satisfying $^{9}$

$$
a \leq \square \diamond a,
$$

which can be rewritten into a quasi-equation of rank 0,1 as:

$$
a \leq \neg \diamond a^{\prime} \text { implies } a^{\prime} \leq \neg \diamond a \text {. }
$$

Total KB algebras are dual to frames with a symmetric relation. In the partial algebra setting, a q-frame $(X, \sim, R)$ corresponds to a partial $\mathrm{KB}$ algebra iff it satisfies, for all $x, y \in X$,

$$
\text { if } x R y \text { then there exists } y^{\prime} \sim y \text { such that } y^{\prime} R x \text {. }
$$

The dual of the functor $F_{\mathbf{K B}}$ may be described as

$$
G_{\mathbf{K B}}(X, \sim, R)=\left(\{(x, T) \in X \times \mathcal{P}(X) \mid R[x]=[T] \sim, \forall y \in T . y R x\},{ }_{1}, R_{\ni}\right) .
$$

Again, the projection map $G_{\mathbf{K B}}(X, \sim, R) \rightarrow(X, \sim, R)$ is surjective for any $\mathrm{KB}$ q-frame and the approximating chain of the free $\mathrm{KB}$ algebra over a set $P$ may be described dually by

\footnotetext{
${ }^{9}$ As usual in modal logic, $\square b$ is shorthand for $\neg \diamond \neg b$.
} 
repeated application of the functor $G_{\mathbf{K B}}$ to

$$
\left(X_{P} \times \mathcal{P}\left(X_{P}\right),=_{1}, R_{\ni}\right)
$$

which happens to be a KB q-frame already.

A final interesting example is the class of $\mathrm{K} 5$ algebras, i.e., the class of modal algebras satisfying

$$
\diamond a \leq \square \diamond a
$$

These are the modal algebras dual to so called Euclidean frames. A q-frame $(X, \sim, R)$ is dual to a partial $\mathrm{K} 5$ algebra iff it satisfies, for all $x, y, z \in X$,

$$
\text { if } z R x \text { and } z R y \text {, then there exists } x^{\prime} \sim x \text { such that } x^{\prime} R y \text {. }
$$

In this case the projection map $\pi_{X}: G_{\mathbf{K 5}}(X, \sim, R) \rightarrow(X, \sim, R)$ is not always surjective, as may be seen by considering the $\mathrm{K} 5 \mathrm{q}$-frame depicted in the following figure.

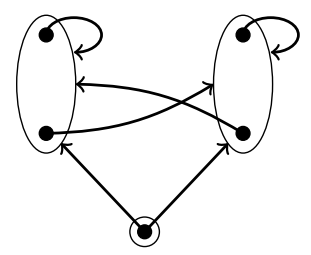

However, for a finite set $P$, the q-frame $\left(X_{P} \times \mathcal{P}\left(X_{P}\right),=_{1}, R_{\ni}\right)$ is a $\mathrm{K} 5 \mathrm{q}$-frame and repeated application of $G_{\mathbf{K} 5}$ yields a chain with surjective projective maps. In fact, from the fourth step onwards this chain is constant, which implies that the finitely generated K5 algebras are finite.

The examples mentioned in this section may be compared with the finite models in [13], which are similar, but were obtained independently and via completely different methods. We believe a comparison of our results with those in [13] would be interesting future work. ${ }^{10}$

\section{Partial algebras for a functor}

In this section, we will show how our construction encompasses the following result of Kurz and Bezhanishvili [2]. If a modal logic $\mathcal{L}$ is axiomatized by pure rank 1 axioms, then, by results in [11], its class of algebras $\mathbf{V}_{\mathcal{L}}$ consists exactly of the algebras for a functor $L$ on the category of Boolean algebras. It was shown in [2] that the approximating chain for the free $\mathbf{V}_{\mathcal{L}}$ algebra can be obtained by a uniform step-by-step construction using the functor $L$.

We will sketch the translation of their result into our setting. In order to do so we define the category pLA of partial L-algebras for a given functor $L$ on Boolean algebras, as follows.

\footnotetext{
${ }^{10}$ We thank Tadeusz Litak for pointing us to this reference, and for interesting discussions on this line of work.
} 
Definition 7.1. Let $L: \mathbf{B A} \rightarrow \mathbf{B A}$ be a functor. ${ }^{11}$ A partial $\mathbf{L}$-algebra is a tuple $(B, A, i, \alpha)$, where $A, B$ are Boolean algebras, $i: A \dashv B$ is an embedding, and $\alpha: L A \rightarrow B$ is a Boolean homomorphism.

A pLA morphism from $\left(B_{1}, A_{1}, i_{1}, \alpha_{1}\right)$ to $\left(B_{2}, A_{2}, i_{2}, \alpha_{2}\right)$ is a pair $\left(f, f^{\prime}\right)$, with $f: B_{1} \rightarrow B_{2}$ and $f^{\prime}: A_{1} \rightarrow A_{2}$ homomorphisms such that the following diagrams commute:
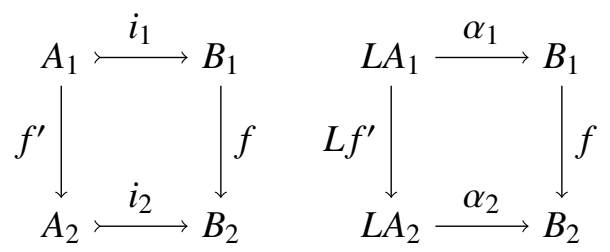

We denote the category of partial $L$-algebras by pLA, and the full subcategory of partial $L$-algebras for which $B$ is finite by $\mathbf{p} \mathbf{L} \mathbf{A}_{\omega}$. We call a partial $L$-algebra $(B, A, i, \alpha)$ total if $i$ is an isomorphism, and denote the full subcategory of total $L$-algebras by tLA.

Remark 7.2. Note in particular that for the functor $L=F_{\mathbf{B A}}^{\vee}$, the category $\mathbf{p L A}$ is equivalent to the category pMA of partial modal algebras. Hence, the free algebra for the variety $\mathbf{V}_{\mathbf{K}}$ associated with the basic modal logic $\mathbf{K}$ can now also be constructed using the method outlined in this section. More generally, if $L$ is a functor for a logic whose variety $\mathbf{V}$ is defined by rank 1 equations, then the category $\mathbf{p V}$ is equivalent to the category $\mathbf{p L A}$.

We now have a functor $F_{L}$ on the category of finite partial $L$-algebras, as follows. Given a finite partial $L$-algebra $(B, A, i, \alpha)$, let $\left(F_{L} B, j, \beta\right)$ be the following pushout in BA:

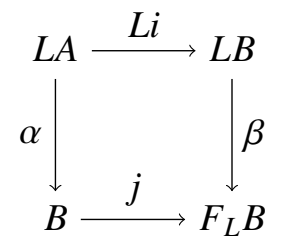

Then $\left(F_{L} B, B, j, \beta\right)$ is a partial $L$-algebra, by the following lemma.

Lemma 7.3. The map $j$ is an embedding.

Proof. Since $i$ has a left inverse, $L i$ has a left inverse. By the universal property of the pushout, we obtain a left inverse for $j$.

To define $F_{L}$ on morphisms, let $\left(f, f^{\prime}\right):\left(B_{1}, A_{1}, i_{1}, \alpha_{1}\right) \rightarrow\left(B_{2}, A_{2}, i_{2}, \alpha_{2}\right)$ be a pLA morphism. We define the pair $F_{L}\left(f, f^{\prime}\right):=\left(g, g^{\prime}\right):\left(F_{L} B_{1}, B_{1}, j_{1}, \beta_{1}\right) \rightarrow\left(F_{L} B_{2}, B_{2}, j_{2}, \beta_{2}\right)$ by letting $g^{\prime}:=f$, and defining $g: F_{L} B_{1} \rightarrow F_{L} B_{2}$ to be the unique map, given by the universal property of the pushout $F_{L} B_{1}$, which factors the following commutative diagram:

\footnotetext{
${ }^{11}$ Throughout this section, we will assume that $L$ sends finite Boolean algebras to finite Boolean algebras
} 


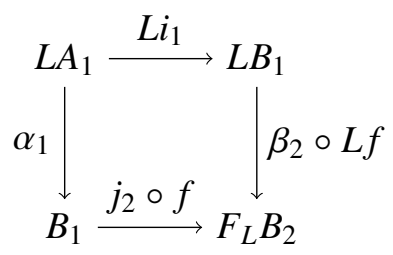

Remark 7.4. Suppose $\mathbf{V}_{\mathcal{E}}$ is a variety of partial modal algebras defined by pure rank 1 equations. Let $L$ be the functor associated with this variety. Then, in this special case, the free image-total functor $F_{\mathcal{E}}$ from Definition 3.11 and the functor $F_{L}$ coincide. (Here, we identify the categories $\mathbf{p V}$ and $\mathbf{p L A}$, which are equivalent by Remark 7.2).

We will now show that repeated application of the functor $F_{L}$ yields essentially the same chain of algebras as defined by Kurz and Bezhanishvili [2], and hence, by their result, it is the approximating chain of the free $\mathbf{V}_{\mathcal{L}}$ algebra.

Recall that Kurz and Bezhanishvili constructed a chain of Boolean algebras by defining, starting from a given Boolean algebra $C_{0}$,

$$
C_{n+1}:=C_{0}+L\left(C_{n}\right)
$$

and letting $e_{0}: C_{0} \rightarrow C_{1}=C_{0}+L C_{0}$ be the inclusion map into the first summand, and then inductively defining $e_{n+1}:=\operatorname{id}_{C_{0}}+L e_{n}: C_{0}+L C_{n} \rightarrow C_{0}+L C_{n+1}$.

Now, to obtain this chain of Boolean algebras $\left(C_{n}\right)_{n \geq 0}$ in our setting, let $C_{0}$ be a finite Boolean algebra. We associate to it the partial $L$-algebra $B_{1}:=\left(C_{0}+L C_{0}, C_{0}, \kappa_{1}, \kappa_{2}\right)$, where $\kappa_{1}: C_{0} \rightarrow C_{0}+L C_{0}$ and $\kappa_{2}: L C_{0} \rightarrow C_{0}+L C_{0}$ are the coprojection maps (note that, in the category BA, both $\kappa_{1}$ and $\kappa_{2}$ are monomorphisms). Now simply define a chain of partial $L$-algebras by putting, for $n \geq 1$,

$$
B_{n+1}:=F_{L}\left(B_{n}\right)
$$

Proposition 7.5. For each $n \geq 0$, the partial L-algebra $B_{n+1}=F_{L} B_{n}$ is (isomorphic to) $\left(C_{n+1}, C_{n}, e_{n}, \kappa_{2}^{n}\right)$, where $\left(e_{n}: C_{n} \rightarrow C_{n+1}\right)_{n \geq 0}$ is the chain defined in (9) above, and $\kappa_{2}^{n}$ : $L C_{n} \rightarrow C_{n+1}$ is the coproduct map into the second coordinate.

Proof. For $n=0$, this is true by definition of $B_{1}$ and $e_{0}$. For $n \geq 1$, using induction, this amounts to showing that the following is a pushout diagram:

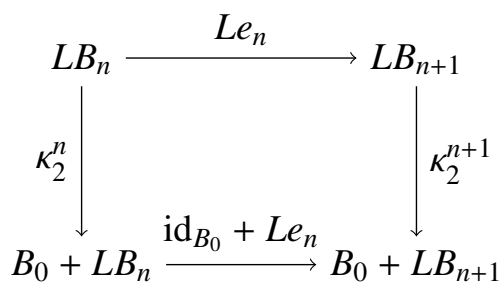

which can be done easily, either using duality for Boolean algebras or directly. 
Theorem 4.2 of [2] states that the colimit of the chain $\left(C_{n}\right)_{n \geq 0}$ yields the free $\mathbf{V}_{\mathcal{L}}$ algebra. We have proved here that this chain can also be obtained by repeated application of the functor $F_{L}$, and thus also yields the free $\mathbf{V}_{\mathcal{L}}$ algebra. By Remark 7.4, it now follows that our construction of a free image-total functor $F_{\mathcal{E}}$ in Section 3 encompasses the known result from [2].

\section{Future work}

We have seen in this paper that, for any variety $\mathbf{V}_{\mathcal{E}}$ which is axiomatized by a set $\mathcal{E}$ of rank 0-1 equations, the free $\mathbf{V}_{\mathcal{E}}$ algebra can be built by repeatedly applying the functor $F_{\mathcal{E}}$. As remarked in the introduction, this method for building the free algebra is particularly useful for applications in the case where the transformation $\eta: 1 \rightarrow F_{\mathcal{E}}$ is pointwise injective. In all examples that were considered here and in the literature, notably the classes axiomatized by pure rank 1 equations and the class of T modal algebras, $\eta$ is indeed pointwise injective. The conjecture that $\eta$ is injective for any set of rank 0-1 equations remains open, and is an important next step in this research project.

In the case where $\mathcal{E}$ is a set of rank 0-1 quasi-equations, repeated application of the functor $F_{\mathcal{E}}$ yields the free algebra in the colimit if the condition that the maps $\eta_{B_{n}}$ are injective throughout the chain is satisfied. Therefore, the more general question of when this condition holds is also an interesting topic for future work. We have shown that, for partial S4 algebra and KB algebras, one always gets embeddings. We do not get embeddings in general for partial K5 algebras, however, the maps arising in our construction of the free K5 algebras over a finite set are all embeddings, hence, the method does apply. It follows from the existence of non-decidable logics that we cannot hope that, for every set of quasi-equations, the maps $\eta_{B_{n}}$ in the construction of the free algebra are embeddings. We conjecture that there even exist decidable logics for which the maps $\eta_{B_{n}}$ are not all embeddings. Finding examples of such logics is left for future work. An interesting example to study would be the class of GL modal algebras, which correspond to provability logic. Furthermore, our method may readily be extended to multimodal algebras. This provides a new supply of examples (with relatively simple axiomatizations) which may lead to new insights.

Finally, we have remarked that, if we have an approximating chain for a quasi-variety $\mathbf{V}_{\mathcal{E}}$ in which all maps $\eta_{B_{n}}$ are injective, then we get normal forms for the logic to which the quasivariety $\mathbf{V}_{\mathcal{E}}$ is associated. It is therefore natural to ask whether normal forms always arise in this way, i.e., if a logic has normal forms, must the approximating chain arising from the functor $F_{\mathcal{E}}$ then necessarily be injective? If this is true, then it would entail that the method outlined in this paper provides an exhaustive search for normal forms, in the sense that if a logic has normal forms, then the method outlined in this paper will yield them. 


\section{References}

[1] Nick Bezhanishvili and Mai Gehrke, Finitely generated free Heyting algebras via Birkhoff duality and coalgebra, Logical Methods in Computer Science 7 (2011), no. 2, $1-24$.

[2] Nick Bezhanishvili and Alexander Kurz, Free modal algebras: a coalgebraic perspective, in: Algebra and Coalgebra in Computer Science, Lecture Notes in Computer Science, vol. 4624, Springer, 2007, pp. 143-157.

[3] Patrick Blackburn, Maarten de Rijke, and Yde Venema, Modal logic, Cambridge Tracts in Theoretical Computer Science, vol. 53, Cambridge University Press, 2001.

[4] Peter Burmeister, A Model Theoretic Oriented Approach to Partial Algebras, 'Mathematical Research' series, vol. 32, Akademie Verlag, 1986.

[5] Dion Coumans and Sam van Gool, Constructing the Lindenbaum algebra for a logic step-by-step using duality (extended version), (2011), To appear in: Proceedings of $\mathrm{PhDs}$ in Logic, Brussels, 2011, available online at http://www.math.ru.nl/ vangool/coumansvangool17012011.pdf.

[6] Silvio Ghilardi, Free Heyting algebras as bi-Heyting algebras, Math. Rep. Acad. Sci. Canada XVI (1992), no. 6, 240-244.

[7] An algebraic theory of normal forms, Annals of Pure and Applied Logic 71 (1995), 189-245.

[8] _ Continuity, Freeness, and Filtrations, Journal of Applied Non Classical Logics 20 (2010), no. 3, 193-217.

[9] Robert Goldblatt, Varieties of complex algebras, Annals of Pure and Applied Logic 44 (1989), 173-242.

[10] George Grätzer, Universal Algebra, 2nd ed., Springer, 1979.

[11] A. Kurz and J. Rosicky, Strongly complete logics for coalgebras, (2006), available online at http://www.cs.le.ac.uk/people/akurz/Papers/KR/strcompl.pdf.

[12] E. G. Manes, Algebraic theories, Graduate Texts in Mathematics, vol. 26, SpringerVerlag, 1976.

[13] Lawrence S. Moss, Finite models constructed from canonical formulas, Journal of Philosophical Logic 36 (2007), no. 6, pp. 605-640.

[14] Yde Venema, Algebras and Coalgebras, Handbook of Modal Logic (Patrick Blackburn, Johan van Benthem, and Frank Wolter, eds.), Elsevier, 2007, pp. 331-426. 